This is a postprint version of the following published document:

Rodríguez-Martínez, J.A.; Molinari, A.; Zaera, R.; Vadillo, G.; FernándezSáez, J. The critical neck spacing in ductile plates subjected to dynamic biaxial loading: on the interplay between loading path and inertia effects. International Journal of Solids and Structures, Vol. 108, Pp. 74-84, 1 March 2017

DOI: https://doi.org/10.1016/i.iisolstr.2016.11.007

(C) 2016 Elsevier Ltd. All rights reserved.

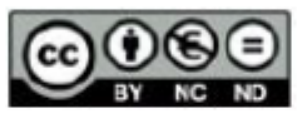

This work is licensed under a Creative Commons Attribution-NonCommercialNoDerivatives 4.0 International License. 


\title{
The critical neck spacing in ductile plates subjected to dynamic biaxial loading: On the interplay between loading path and inertia effects
}

\author{
J.A. Rodríguez-Martínez ${ }^{\mathrm{a}, \mathrm{b}}$, A. Molinarib ${ }^{\mathrm{b}, *}$, R. Zaera ${ }^{\mathrm{a}}$, G. Vadillo ${ }^{\mathrm{a}}$, J. Fernández-Sáez ${ }^{\mathrm{a}}$ \\ ${ }^{a}$ Department of Continuum Mechanics and Structural Analysis. University Carlos III of Madrid. Avda. \\ de la Universidad, 30. 28911 Leganés, Madrid, Spain \\ ${ }^{b}$ Laboratoire d'Etudes des Microstructures et de Mécanique des Matériaux LEM3, UMR CNRS 7239, \\ Université de Lorraine, Ile du Saulcy, 57045 Metz cedex 1,France
}

\begin{abstract}
In this work we have investigated the emergence of a critical wavelength which characterizes the localization pattern in ductile plates subjected to dynamic biaxial loading. For that task we have used a linear stability analysis and finite element calculations. The linear stability analysis follows the 2D approach developed by Zaera et al. (2015) which includes specific features to account for inertia and stress triaxiality effects inside the necking. Two different finite element models are built: (1) a unitary cell model in which the localization is favoured by a sinusoidal geometrical perturbation and (2) a plate with constant cross section which allows to assess the collective behaviour of multiple necks. A wide spectrum of loading paths which range from plane strain to (almost) biaxial stretching has been explored. We have demonstrated that, if inertia plays a dominant role in the loading process, the influence of geometrical perturbations in the necking inception is substantially reduced and the necking pattern shows a deterministic nature. The deterministic nature is directly connected to the emergence of a critical wavelength which characterizes the neck spacing at high strain rates. This critical wavelength increases (i.e. the neck spacing increases) and becomes less prevailing (i.e. the necking pattern becomes less uniform) as we move away from plane strain to biaxial stretching. This is a key outcome of our investigation that, from the authors' knowledge, has not been previously reported in the literature.
\end{abstract}

Keywords:

\footnotetext{
*Corresponding author. Tel. $\quad+33$ 387315369; Fax: +33 387315366. E-mail address: alain.molinari@univ-lorraine.fr
} 
Dynamic biaxial loading, Inertia, Necking, Critical wavelength, Localization pattern

\section{Introduction}

The problem of flow localization and fragmentation in ductile materials subjected to dynamic loading has attracted the interest of the Solid Mechanics community for almost a century. The starting point of this research was the work of Mott (1947) who, moved by the military concerns of that time, developed during the latter part of World War II a theoretical framework to describe the fragmentation of bodies subjected to intense impulsive loads. Specifically, the attention of Mott was focused on the fragmentation resulting from the explosive rupture of cylindrical cases. Seventy years have passed since the pioneering research of Mott was published and, in Grady's words (Grady, 2002), the fragmentation of hollow metal shells subjected to rapid expansion by impulsive internal pressure loading continues to be a problem of both practical and intellectual interest. Grady's statement becomes apparent if one considers the number of works that have been published over the last years on this specific topic (e.g. Sørensen and Freund (2000); Becker (2002); Guduru and Freund (2002); Rusinek and Zaera (2007); Vadillo et al. (2012); De Vuyst and Vignjevic (2013)).

Within this context, the recent and very significant experimental research relied on the dynamic radial expansion of axially symmetric structures like tubes (Goto et al., 2008; Hiroe et al., 2008; Zhang and Ravi-Chandar, 2010) and hemispheres (Mercier et al., 2010). The symmetry of these shell structures nearly eliminates the effects of wave propagation before flow localization, which facilitates the interpretation of the experimental findings. Thus, the shell uniformly stretches during loading until homogeneous deformation fails at large strain, leading to flow localization in the form of multiple necks and subsequent fragmentation. The experimental research have yielded two fundamental observations:

1. As the loading rate applied to the shell increases, the apparent ductility of the structure increases.

2. As the loading rate applied to the shell increases, the number of fragments increases.

To this day, there is a lack of consensus in the determination of the physical phenomena which reside behind these two experimental observations. Particularly, the identification 
of the specific mechanisms which control the fragmentation pattern is a critical concern that has been debated for many years.

On the one hand we should mention the statistically-based theories, derived from the (aforementioned) seminal work of Mott (1947), which consider that fragmentation proceeds through the random spatial and temporal occurrence of fractures resulting in a distribution in fragment lengths. These theories, significantly moved forward over the last three decades by Grady and co-workers (Grady, 1981; Kipp and Grady, 1985; Grady and Olsen, 2003), attribute the distribution of fragment sizes to statistical variability in the strain to failure of the material and obtain this distribution through an estimate of the propagation of the unloading or release waves from each fracture event. Very recently, this theoretical framework has been taken by Ravi-Chandar and Triantafyllidis (2015) in order to show that the localization and failure patterns of the structure may be dictated by the distribution of intrinsic material or/and geometrical defects.

On the other hand we should mention the linear stability theories, derived from the pioneering papers of Fressengeas and Molinari $(1985,1994)$, which consider that the combination of stress multiaxiality effects and inertia leads to the promotion of some specific wavelengths which characterize the localization and fragmentation patterns at high strain rates (Mercier and Molinari, 2003, 2004; Mercier et al., 2010; Rodríguez-Martínez et al., 2013a,b; El Maï et al., 2014). In such a sense, this approach argues for the inclusion of a deterministic component within the fragmentation mechanisms. Nevertheless, this idea has been frequently disregarded in the classical statistical theories.

A main goal of this paper is to show that the deterministic approach which follows from the stability analysis theories actually captures key features of the necking inception at high strain rates. While defects play an important role in the localization and fragmentation process, it seems that they are not the leading factor which controls the fragmentation pattern of ductile metallic shells subjected to very high loading rates. In this paper we rely on finite element calculations and a linear stability analysis to show that, at high strain rates, there are selected wavelengths that characterize the localization and fragmentation patterns. We demonstrate that the role played by these selected wavelengths in the neck spacing and the fragments size is more significant as the contribution of inertia in the 
process of flow localization increases. In addition, we present some results that confirm that if inertia is the main controlling factor of the loading process, the geometrical defects may not dictate the neck spacing and the fragments size. A salient feature of this investigation, which generalizes the results of Xue et al. (2008), is that all these outcomes have been proven for a wide range of loading paths ranging from plane strain to (almost) biaxial stretching.

\section{Problem formulation}

We consider a rectangular sheet of initial thickness $h^{0}$ and edges of initial length $L_{X}^{0}$ and $L_{Y}^{0}$, see Fig. 1. Capital letters representing coordinates, displacements, velocities or accelerations will be used for the Lagrangian frame, and lower-case letters for the Eulerian frame. The sheet is subjected to constant and opposed velocities on opposed sides and therefore directions $X$ and $Y$ are of overall principal strain rates. During the stage of homogeneous deformation, the initial strain rates $\dot{\varepsilon}_{X}^{0}$ and $\dot{\varepsilon}_{Y}^{0}$ fully define the kinematics. Note that our attention is limited to loading paths for which $\chi_{\dot{\varepsilon}}=\dot{\varepsilon}_{Y}^{0} / \dot{\varepsilon}_{X}^{0} \geq 0$.

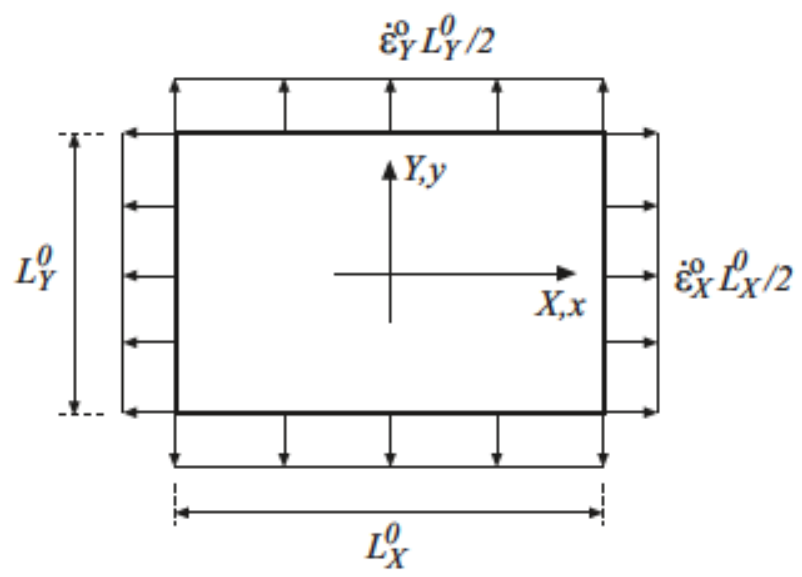

$\{X, Y\}$ : principal strain rate directions

Figure 1: Sheet domain and principal axes of deformation.

The fundamental solution $\mathbb{S}(X, Y, t)$ of the previous problem is obtained by integration of the corresponding governing equations (see Zaera et al. (2015) for details) satisfying the following initial 


$$
V_{X}(X, Y, Z, 0)=\dot{\varepsilon}_{X}^{0} X ; \quad V_{Y}(X, Y, Z, 0)=\dot{\varepsilon}_{Y}^{0} Y ; \quad V_{Z}(X, Y, Z, 0)=-\left(\dot{\varepsilon}_{X}^{0}+\dot{\varepsilon}_{Y}^{0}\right) Z
$$

and boundary conditions

$$
\begin{aligned}
& V_{X}\left(L_{X}^{0} / 2, Y, Z, t\right)=-V_{X}\left(-L_{X}^{0} / 2, Y, Z, t\right)=\dot{\varepsilon}_{X}^{0} L_{X}^{0} / 2 \\
& V_{Y}\left(X, L_{Y}^{0} / 2, Z, t\right)=-V_{Y}\left(X,-L_{Y}^{0} / 2, Z, t\right)=\dot{\varepsilon}_{Y}^{0} L_{Y}^{0} / 2
\end{aligned}
$$

The material behaviour is described with a rate dependent constitutive model based on the standard principles of Huber-Mises plasticity: hypoelastic behaviour, additive decomposition of the rate of deformation tensor, isotropic hardening, associated flow rule and plastic power equivalence

$$
\begin{gathered}
\sigma^{\nabla}=\mathbf{C}: \mathbf{d}^{e}=\mathbf{C}:\left(\mathbf{d}-\mathbf{d}^{p}\right) \\
\Psi=\bar{\sigma}-\sigma_{Y}=0 \\
\mathbf{d}^{p}=\frac{\partial \Psi}{\partial \sigma} \dot{\varepsilon}^{p}=\frac{3 \mathrm{~s}}{2 \bar{\sigma}} \dot{\varepsilon}^{p}
\end{gathered}
$$

where $\sigma^{\nabla}$ is an objective derivative of the Cauchy stress tensor, $\mathbf{d}, \mathbf{d}^{e}$ and $\mathbf{d}^{p}$ are the total, elastic and plastic rate of deformation tensors respectively, $\mathbf{C}$ is the Hooke tensor for isotropic elasticity (defined by the Young modulus $E$ and the Poisson ratio $\nu$ ), $\Psi$ the yield function, $\bar{\sigma}$ the equivalent stress, $\sigma_{Y}$ is the flow stress, $\mathbf{s}$ the deviatoric stress tensor and $\dot{\varepsilon}^{p}$ is the equivalent plastic strain rate.

Following the work of Rodríguez-Martínez et al. (2013a), the flow stress is given as a function of the equivalent plastic strain rate $\dot{\varepsilon}^{p}$ through the following power-type relation 


$$
\sigma_{Y}=\sigma^{0}\left(\frac{\dot{\bar{\varepsilon}}^{p}}{\dot{\bar{\varepsilon}}_{r e f}}\right)^{m}
$$

Conventional material constants, elastic parameters and parameters related to the flow stress are given in Table 1.

\begin{tabular}{ccc}
\hline Symbol & Property and units & Value \\
\hline$\rho^{0}$ & Initial density $\left(\mathrm{kg} / \mathrm{m}^{3}\right)$ & 7800 \\
\hline$E$ & Young modulus $(\mathrm{GPa})$ & 200 \\
$\nu$ & Poisson ratio & 0.33 \\
\hline$\sigma^{0}$ & Reference flow stress $(\mathrm{MPa}), \mathrm{Eq} .(6)$ & 500 \\
$\dot{\bar{\varepsilon}}_{r e f}$ & Reference strain rate $\left(\mathrm{s}^{-1}\right)$, Eq. (6) & 1000 \\
$m$ & Strain rate sensitivity exponent, Eq. (6) & 0.01 \\
\hline
\end{tabular}

Table 1: Conventional material constants, elastic parameters and parameters related to the flow stress as taken from Rodríguez-Martínez et al. (2013a).

No doubt, more sophisticated constitutive descriptions could be used to model the material behaviour (see e.g. Nemat-Nasser and Guo (2000); Rusinek and Klepaczko (2001); Molinari and Ravichandran (2005); Voyiadjis and Abed (2006)). Nevertheless, we claim that this simple constitutive model is sufficient to uncover the dominant factors which control the emergence of a critical wavelength responsible for the fragmentation of ductile plates subjected to high strain rates. Moreover, as pointed out by Rodríguez-Martínez et al. (2013a), this simple constitutive law is well suited to carry out critical comparisons between finite elements calculations and stability analysis, see section 8 .

\section{Linear stability analysis}

The linear stability analysis follows the approach developed in Zaera et al. (2015), which is based on previous works of Dudzinski and Molinari $(1988,1991)$ and includes specific features to account for inertia and stress triaxiality effects inside the necking. The 
perturbation is imposed on lines $X=$ constant (see Fig. 1) since this is the orientation naturally selected by the material to trigger a neck (critical direction of perturbation). Let $\mathbb{S}^{1}$ be the corresponding value of the fundamental solution at time $t^{1}$, when a small perturbation $\delta \mathbb{S}$ given by

$$
\delta \mathbb{S}\left(X, t ; t^{1}\right)=\delta \mathbb{S}^{1} e^{i \xi^{\mathscr{S}} X} e^{\eta\left(t-t^{1}\right)}
$$

is imposed over the fundamental solution. In previous expression $\delta \mathbb{S}^{1}$ is the perturbation amplitude, $\xi^{\mathscr{L}}$ the wavenumber in the Lagrangian configuration and $\eta$ the growth rate of the perturbation at time $t^{1}$. The physical solution is the real part of $\mathbb{S}=\mathbb{S}^{1}+\delta \mathbb{S}$ with $|\delta \mathbb{S}| \ll\left|\mathbb{S}^{1}\right|$. By substituting $\mathbb{S}$ into the governing equations and retaining only first-order terms, linearised equations are obtained. A non-trivial solution for $\delta \mathbb{S}^{1}$ can be derived only if the determinant of the system of linear algebraic equations is equal to zero. This condition is found to be a polynomial in $\eta$ which gives, for a certain value of the time at perturbation $t^{1}$, the value of $\eta$ as a function of the wavenumber in the material description $\xi^{\mathscr{L}}$. The requisite for unstable growth of $\delta \mathbb{S}$ is given by the condition $\operatorname{Re}(\eta)>0$. The root of the quoted polynomial which is real and positive $\eta^{+}$represents the unstable growth. Note that, according to Rodríguez-Martínez et al. (2013b, 2015), the perturbation growth $\eta^{+}$is assumed to represent the very first stages at which the plastic flow deviates from the background deformation (the very first stages of diffuse necking). Moreover, imposing the condition for maximum perturbation growth $\partial \eta^{+} / \partial \xi^{\mathscr{L}}=0$, the critical wavenumber $\xi_{c}^{\mathscr{L}}$ and the critical perturbation growth $\eta_{c}^{+}$are determined.

A full description of the linear perturbation analysis and of its salient features can be found in Zaera et al. (2015).

\section{Finite element models}

This section describes the features of the finite element models built to simulate necking localization in ductile plates subjected to dynamic biaxial loading. The numerical analyses are carried out using the finite element program ABAQUS/Explicit (Simulia, 2013). Two different models are built: (1) a unitary cell model in which the localization is favoured 
by a geometrical perturbation and (2) a entire plate with constant cross section which serves to asses the collective behaviour of multiple necks. We anticipate that the results obtained from these two models allow to derive relevant conclusions about the emergence of a critical wavelength which dictates the neck spacing and the necking pattern at high strain rates.

\subsection{Unitary periodic cell}

Inspired by the computational model proposed by Xue et al. (2008) for assessing the necking localization in an infinite plate under plane strain constraint, a plate subjected to biaxial loading and with geometrical periodic perturbations can be modelled as an array of unitary cells with sinusoidal spatial imperfections.

The reference configuration of the cell is given by the domain $-L_{X}^{0} / 2 \leq X \leq L_{X}^{0} / 2$, $-L_{Y}^{0} / 2 \leq Y \leq L_{Y}^{0} / 2$ and $-h^{0} / 2 \leq Z \leq h^{0} / 2$, with $L_{X}^{0}=L_{Y}^{0}=L^{0}$. As further discussed in forthcoming sections of this paper, the value of $L^{0}$ has been systematically varied (while $h^{0}=2 \mathrm{~mm}$ is fixed) in order to assess the relative contribution of stress multiaxiality effects and inertia in flow localization. The spatial imperfection is defined by the following expression

$$
0 \leq Z \leq h^{0} / 2-\frac{\delta}{2}\left(1+\cos \left(\frac{2 \pi X}{L_{X}^{0}}\right)\right)
$$

where $\delta$ is the amplitude of the perturbation. According to the linear stability analysis outlined in section 3 the perturbation is centred on $X=0$ and aligned with the $Y$ axis, following the critical direction of perturbation for $\chi_{\dot{\varepsilon}} \geq 0$ (recall that our attention is limited to loading cases such that $\chi_{\dot{\varepsilon}} \geq 0$ ). Due to the symmetry of the model, only the $Z>0$ half of the specimen has been analysed (see Fig. 2). The applied initial and boundary conditions are those given in Eqs. (1) and (2). As shown by Rodríguez-Martínez et al. (2013a) and Zaera et al. (2014, 2015), these initial conditions minimize the intervention of stress waves within the specimen during the loading process.

The finite element model is meshed using eight node solid elements, with reduced integration and hourglass control $(C 3 D 8 R)$. The elements have an initial aspect ratio close to $1: 1: 1$ with dimensions $\approx 50 \times 50 \times 50 \mu \mathrm{m}^{3}$. A mesh convergence study has 
been performed, in which the time evolution of different critical output variables, namely stress, strain and necking inception, were compared against different mesh sizes. While we have found some mesh sensitivity in the numerical results, we have checked that it does not affect significantly our results, neither quantitatively nor qualitatively. Our belief is that viscosity and inertia act as potent regularization factors that contribute to the well-possessedness of the problem at hand (Needleman, 1988; Rodríguez-Martínez et al., 2013a).

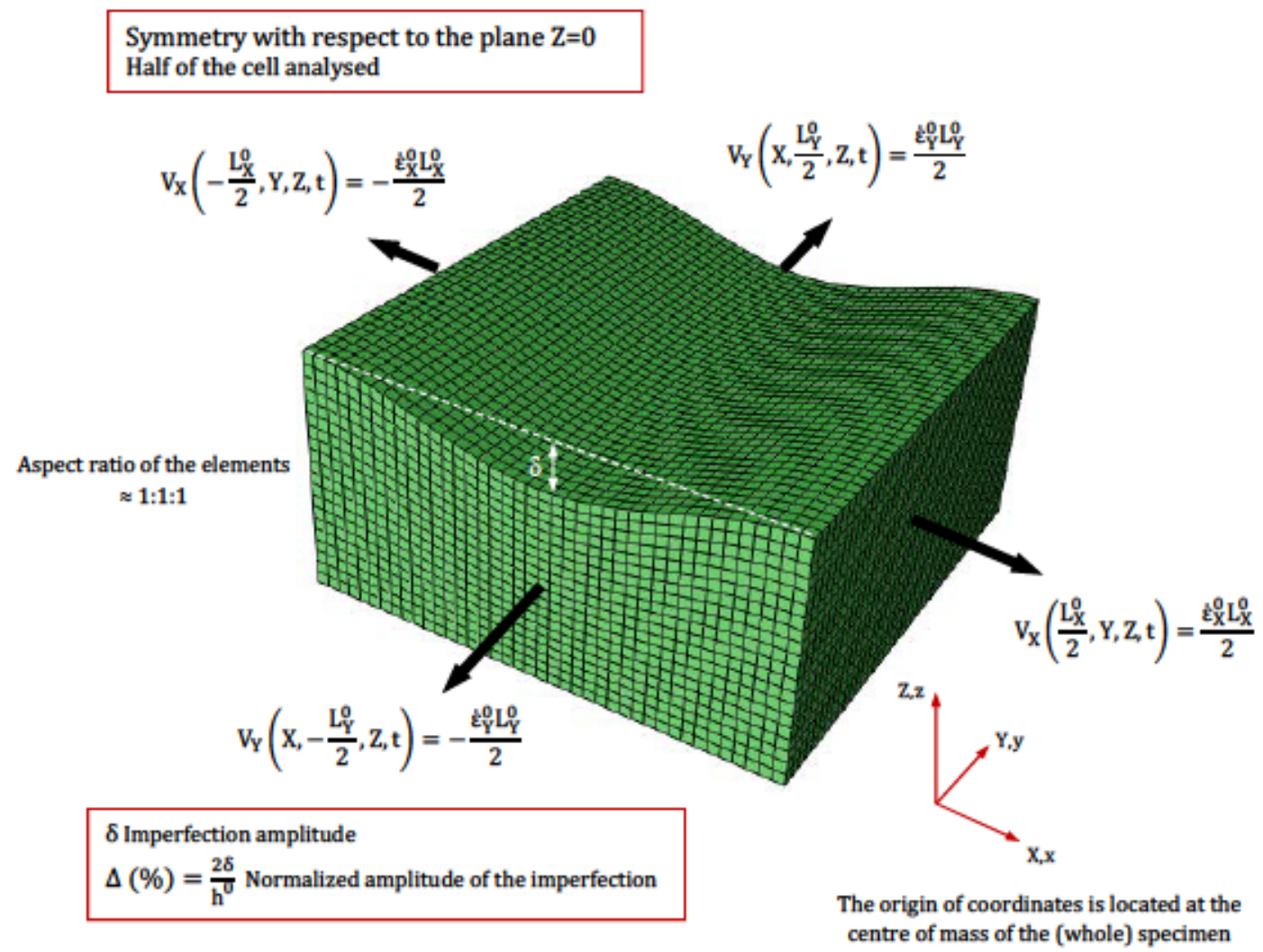

Figure 2: Finite element model. Unitary periodic cell. Mesh and boundary conditions.

\subsection{Entire plate}

Based on the computational model proposed by Zaera et al. (2015) for assessing the collective behaviour and spacing of multiple necks in plates subjected to dynamic biaxial loading, we rely on calculations performed using (entire) plates with constant cross section to explore the interaction between necks at high strain rates.

The reference configuration of the sheet is given by the domain $-L_{X}^{0} / 2 \leq X \leq L_{X}^{0} / 2$, 
$-L_{Y}^{0} / 2 \leq Y \leq L_{Y}^{0} / 2$ and $-h^{0} / 2 \leq Z \leq h^{0} / 2$, with $L_{X}^{0}=L_{Y}^{0}=100 \mathrm{~mm}$ and $h^{0}=2 \mathrm{~mm}$. Only the $Z \geq 0$ half of the specimen has been analysed, see Fig. 3. The initial and boundary conditions applied to the model are those given in Eqs. (1) and (2). These initial conditions minimize the propagation of stress waves in the plate during loading. The sheet has been meshed using a total of $1250000 C 3 D 8 R$ elements, 500 in $X$ and $Y$ directions, and 5 in $Z$ direction. A mesh convergence study has revealed the mesh sensitivity of our numerical results. However, we have checked that the mesh dependence does not affect the conclusions of our investigation. Qualitatively (and quantitatively to a large extend), all the results and trends presented in the paper regarding the necking strain and neck spacing are consistent and independent of the discretization (as long as a sufficiently fine mesh is provided). In absence of geometrical perturbations, localization is triggered by the perturbation of the field variables caused by the discretization of the workpiece and the explicit integration scheme used by the finite element code (Rusinek and Zaera, 2007; Vadillo et al., 2012; Zaera et al., 2015).

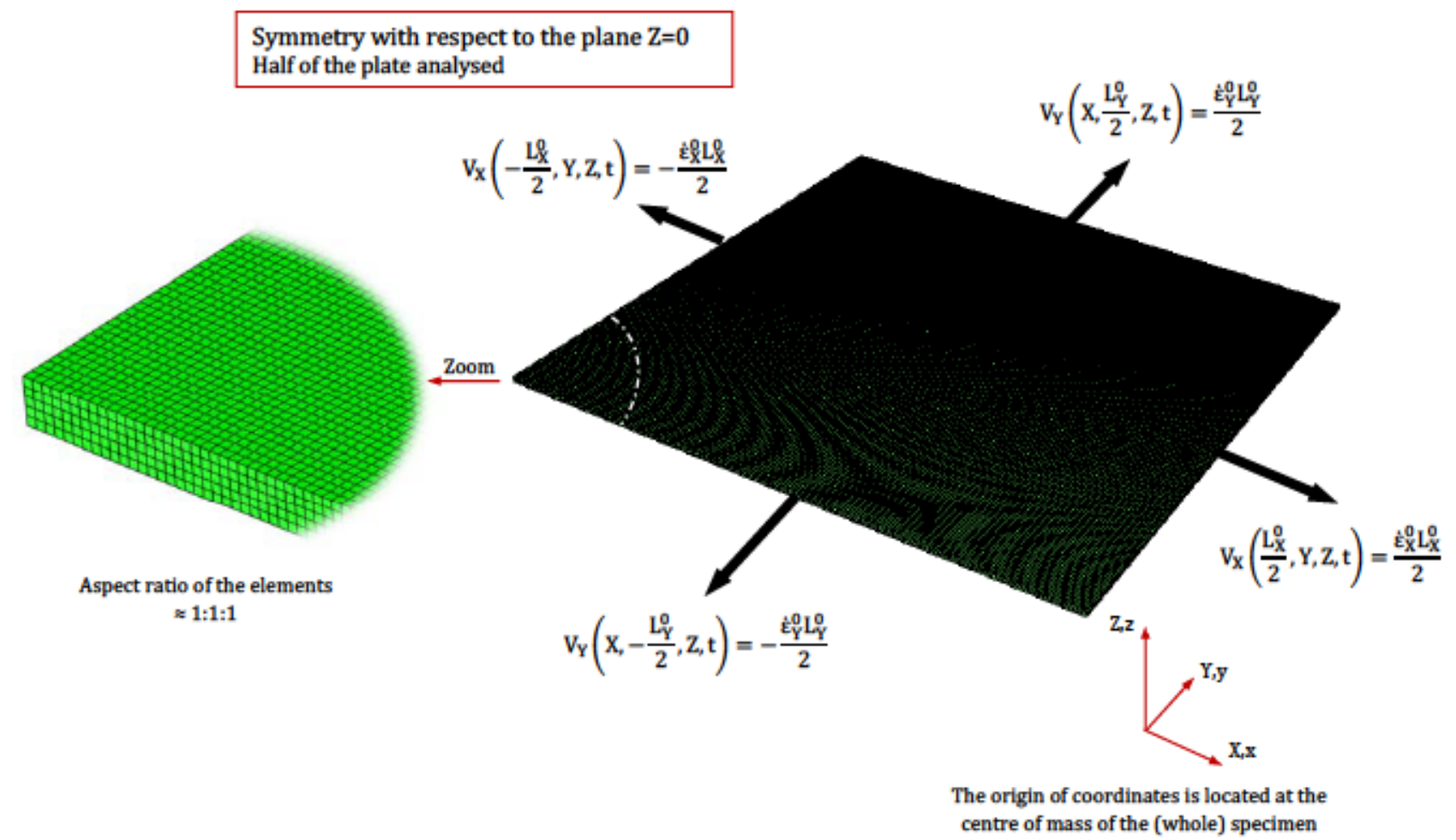

Figure 3: Finite element model. Entire plate. Mesh and boundary conditions. 
The set of equations describing the mechanical behaviour of the material are implemented in the finite element code, considering a large deformation frame, through a user subroutine following the fully implicit integration scheme developed by Zaera and Fernández-Sáez (2006). Note that the lower limit for the strain rate used in the integration algorithm is $10^{-15} \mathrm{~s}^{-1}$.

\section{Analysis and results: the critical wavelength}

In this section we rely on the unitary cell calculations to show the existence of a critical wavelength which plays a key role in the necking and fragmentation patterns of ductile plates subjected to high strain rates.

Fig. 4 shows the localized necking strain $\varepsilon^{\text {neck }}$ versus the initial cell size $L^{0} / h^{0}$ (where $h^{0}=2 \mathrm{~mm}$ ) for $\chi_{\dot{\varepsilon}}=0$ (plane strain), $\Delta=\frac{2 \delta}{h^{0}}=2 \%$ and $\dot{\varepsilon}^{0}=10000 \mathrm{~s}^{-1}$. This combination of loading path, imperfection amplitude and initial loading rate will be considered as the reference configuration for the analyses to be conducted in this paper. According to Xue et al. (2008) and Triantafyllidis and Waldenmyer (2004) the localized necking strain, from now on simply called necking strain, is measured when the condition $\frac{\dot{\varepsilon}^{\text {out }}}{\dot{\varepsilon}^{\text {in }}}=10^{-6}$ is reached, where $\dot{\varepsilon}^{i n}$ and $\dot{\varepsilon}^{\text {out }}$ are the strain rates inside and outside the localized region, respectively. The strain rate inside the localized region $\dot{\varepsilon}^{i n}$ is measured at the centre of the main neck(s) developed in the cell. The strain rate outside the localized region $\dot{\varepsilon}^{\text {out }}$ and the necking strain $\varepsilon^{\text {neck }}$ are measured: (1) at the ends of the sample when the localization takes place in the middle of the cell and (2) at the center of the sample when the localization takes place at the ends of the cell. The necking strain $\varepsilon^{\text {neck }}$ represents the onset of localized necking (this is not the onset of diffuse necking described by the linear perturbation analysis). Note that the large strains attained in the simulations favour the formation of well-developed necks. This is in contrast to more brittle metals which use to show failure by early shear banding or micro-cracking prior to necking inception. In Fig. 4 we observe that the necking strain first decreases when the cell size increases, reaches a minimum and then increases. We have checked that the alternative sine perturbation, minimum section at the ends of the sample, provides the same values of necking strain. On the one hand, the large values of $\varepsilon^{\text {neck }}$ obtained for small $L^{0} / h^{0}$ ratios are caused by the damping effect of stress 
multiaxiality on short wavelengths (Mercier and Molinari, 2003; Zaera et al., 2015). On the other hand, inertia slows down the growth of long wavelengths which leads to the increase of $\varepsilon^{\text {neck }}$ obtained for large $L^{0} / h^{0}$ ratios (Fressengeas and Molinari, 1985, 1994; Mercier and Molinari, 2003; Zaera et al., 2015). Following Zaera et al. (2015), and within the context of the $2 \mathrm{D}$ biaxial loading problem investigated in this paper, the dimensionless number which represents inertia effects is

$$
\tilde{I}=h^{0} \dot{\bar{\varepsilon}} \sqrt{\frac{\rho^{0}}{\sigma^{0}}}
$$

This dimensionless number accounts for the intrinsic material effects that density, sample dimensions, flow stress and loading rate all have on necking inception (Knoche and Needleman, 1993; Mercier and Molinari, 2003, 2004; Zhou et al., 2006).

The combination of the stabilizing aspects of stress multiaxiality on short wavelengths and of inertia on long wavelengths leads to the promotion of an intermediate wavelength which determines the minimum necking strain (Rodríguez-Martínez et al., 2013a). From this point on, the cell size corresponding to the minimum necking strain will be denoted as the critical cell size $\left(L^{0} / h^{0}\right)_{c}$. Recall that lengths are measured in the reference configuration. Note that the value $\left(L^{0} / h^{0}\right)_{c} \approx 3$ reported in Fig. 4 coincides with the critical cell size obtained by Xue et al. (2008) from numerical computations conducted under plane strain conditions. Nevertheless, we have to note that this value of critical cell size varies with the imperfection amplitude and the initial loading rate, as further discussed in sections 6 and 7 .

Fig. 5 shows contours of equivalent plastic strain $\bar{\varepsilon}^{p}$ for different cell sizes. As in Fig. 4 , the reference combination of loading path, imperfection amplitude and initial loading rate is selected. The pictures are taken at times beyond that corresponding to the necking strain, once the plastic strain is fully localized. All the pictures follow the same colour coding such that plastic strains ranging from 0.4 to 1 correlate with a colour scale that comes from blue to red. Plastic strains below 0.4 remain blue and above 1 remain red. In agreement with the results obtained by Xue et al. (2008), we observe that the plastic deformation is concentrated in a smaller (narrower) region as the cell size approaches the critical one. Thus, we identify three distinctive scenarios: 


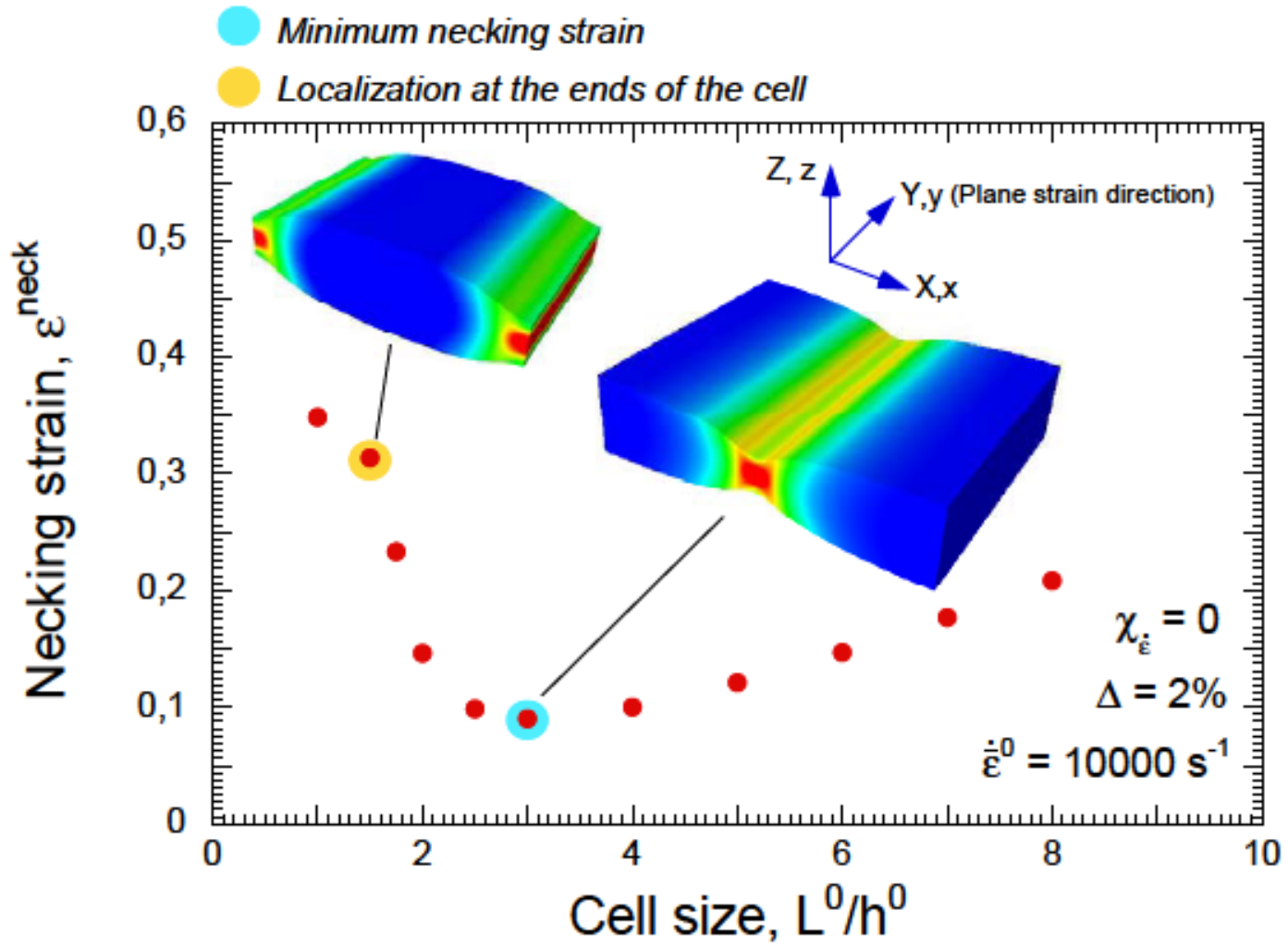

Figure 4: Unitary cell calculations. Necking strain $\varepsilon^{\text {neck }}$ versus initial cell size $L^{0} / h^{0}$ (where $h^{0}=2 m m$ ). Reference configuration: loading path $\chi_{\dot{\varepsilon}}=0$ (plane strain), imperfection amplitude $\Delta=2 \%$ and initial loading rate $\dot{\varepsilon}^{0}=10000 \mathrm{~s}^{-1}$. 
- The cell size is smaller than the critical one $L^{0} / h^{0}<2$ : there is a mild influence of the imperfection on the necking pattern. The plastic strain is spread over a major portion of the cell, the size of the necked region is large. The neck develops at late stages of the deformation process due to stabilizing effects of stress multiaxiality. A large amount of external energy is required to trigger full localization.

The weak influence of the geometrical imperfection on the geometrical pattern is clearly apparent for $L^{0} / h^{0}=1.5$. In this case, the necking does not develop from the initial imperfection but it is located at the ends of the cell. This specific behaviour occurs because the imperfection is smoothed during the loading process up to an extent that it ultimately vanishes (see Rodríguez-Martínez et al. (2013b)). Next, the cell shows a uniform cross section which allows for the inception of the neck either at the center or at the edges. Then, we assume that the fact that the necking occurs in one place (at the center) or another (at the edges, as in this specific case) is determined to a large extent by the spurious disturbances caused by the discretization of the workpiece and the explicit integration scheme used by the code, as discussed in section 4.2. The condition to be fulfilled by the localization pattern is to keep the symmetry enforced by the initial and boundary conditions.

- The cell size is close to the critical one $2 \leq L^{0} / h^{0} \leq 4$ : there is a strong influence of the imperfection on the necking pattern. The plastic strain is mostly concentrated in a small region at the center of the cell. The geometrical imperfection triggers the neck at early stages of the loading process. Little investment of external work is required to reach the stage of full localization.

- The cell size is greater than the critical one $L^{0} / h^{0}>4$ : there is a moderate influence of the imperfection on the necking pattern. The plastic strain is spread over a large area in the center of the plate. Flow localization only takes place at late stages of the loading process.

For $L^{0} / h^{0}=8$, instead of having a single neck, we have three that are symmetrically placed with respect to center of the cell. This makes apparent the mild effect of the imperfection on the necking pattern. As described by Rodríguez-Martínez et al. 
(2013a), this specific behaviour occurs because the material prefers to localize with a shorter wavelength than that determined by the cell size since this requires lower amount of external work to trigger the neck. This conclusion reinforces the idea of the existence of a critical wavelength which plays a significant role in the necking pattern of ductile plates subjected to high strain rates. This role can become so important that it governs the multiple localization process, setting aside those theories which assume that the localization pattern is exclusively controlled by material/geometrical flaws. We have checked that, for cell sizes greater than $L^{0} / h^{0}=8$, the number of necks continues increasing and the localization pattern becomes gradually more (and more) regular. 


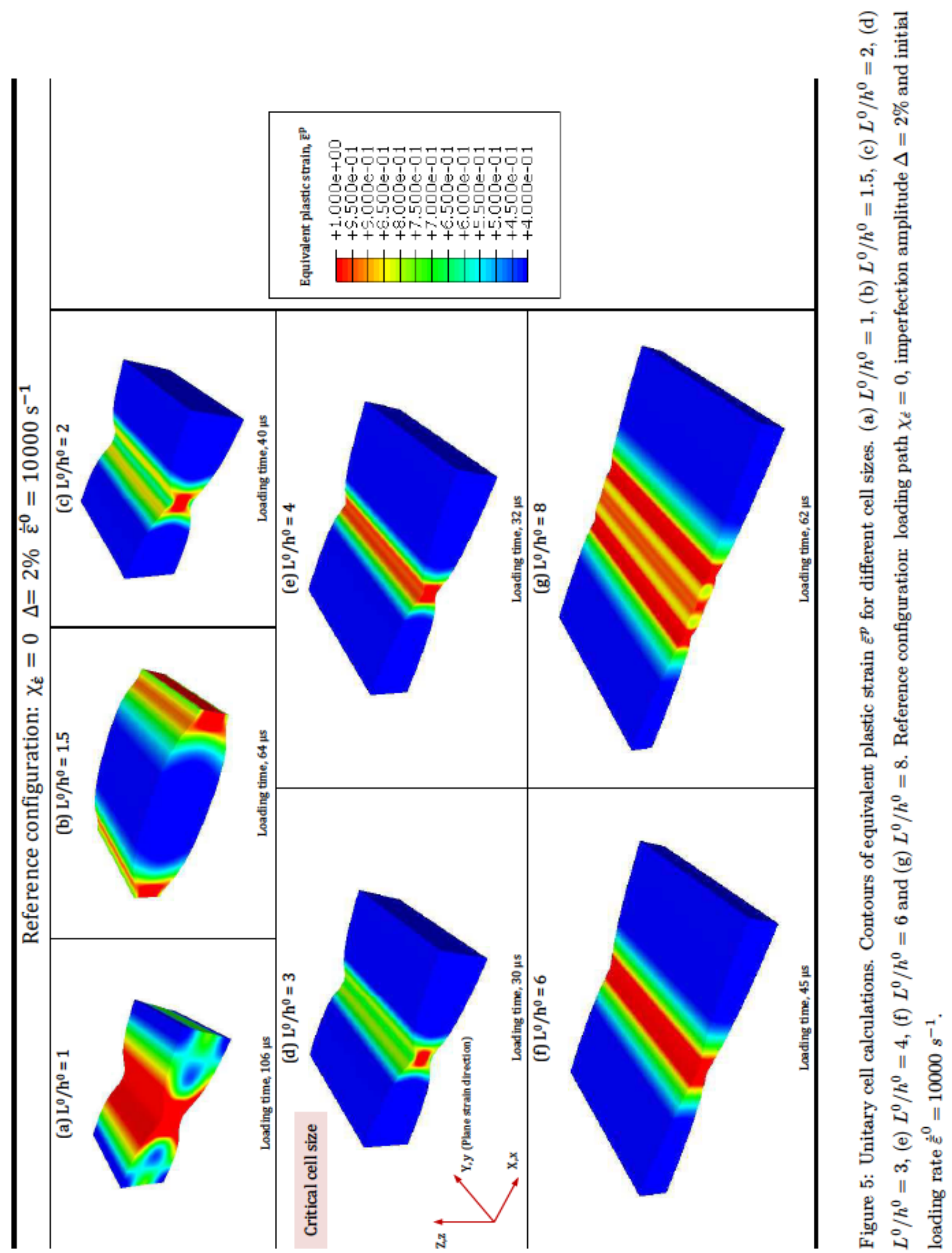




\section{Analysis and results: the effect of imperfection amplitude}

In this section, relying on the unitary cell calculations, we analyse the role played by the imperfection amplitude in the necking process. Fig. 6 shows the necking strain $\varepsilon^{\text {neck }}$ versus the initial cell size $L^{0} / h^{0}$ for different imperfection amplitudes $\Delta=1 \%, \Delta=2 \%$ (reference) and $\Delta=4 \%$. While we have carried out calculations with smaller and larger imperfection amplitudes, they are not shown in the paper for the sake of brevity: the effect of the imperfection on the necking is properly captured by the simulations shown here. It was reported by Rodríguez-Martínez et al. (2013a) that the increase of the imperfection amplitude essentially affects the stress state in the necked region, decreasing the damping effect of stress triaxiality on short wavelengths. As in Figs. 4 and 5, the reference combination of loading path and initial loading rate is selected. As a general rule, we observe a significant decrease in the necking strain with the increase of the imperfection amplitude (there is one exception discussed in the following paragraph). This causes that, as $\Delta$ increases, the minimum of the curve $\varepsilon^{\text {neck }}-L^{0} / h^{0}$ becomes weaker and tends to move to (slightly) larger values of $L^{0} / h^{0}$.

In addition, the strong connection between the imperfection amplitude and the multidimensional character of the stress state in the cell serves to explain the drastic drop in the necking strain obtained in the case of $L^{0} / h^{0}=1$ (smallest cell size) and $\Delta=4 \%$ (greatest imperfection amplitude). For this specific case we do not observe the inception of a neck. The localization process takes the form of a pair of shear bands which are incepted at a early stage of the loading process, see Fig. 7. This dominant shear stress state is caused by the large curvature of the imperfection which results from the combination of a short cell and a large defect amplitude. Shear banding becomes the dominant instability mode. We have checked that the development of the shear bands is suppressed as the imperfection amplitude is reduced.

\section{Analysis and results: the effect of inertia}

In this section the unitary cell computations are used to illustrate the main role played by inertia effects on the emergence of a critical cell size which controls the localization pattern at high strain rates. Fig. 8 shows the necking strain $\varepsilon^{\text {neck }}$ versus the initial cell 


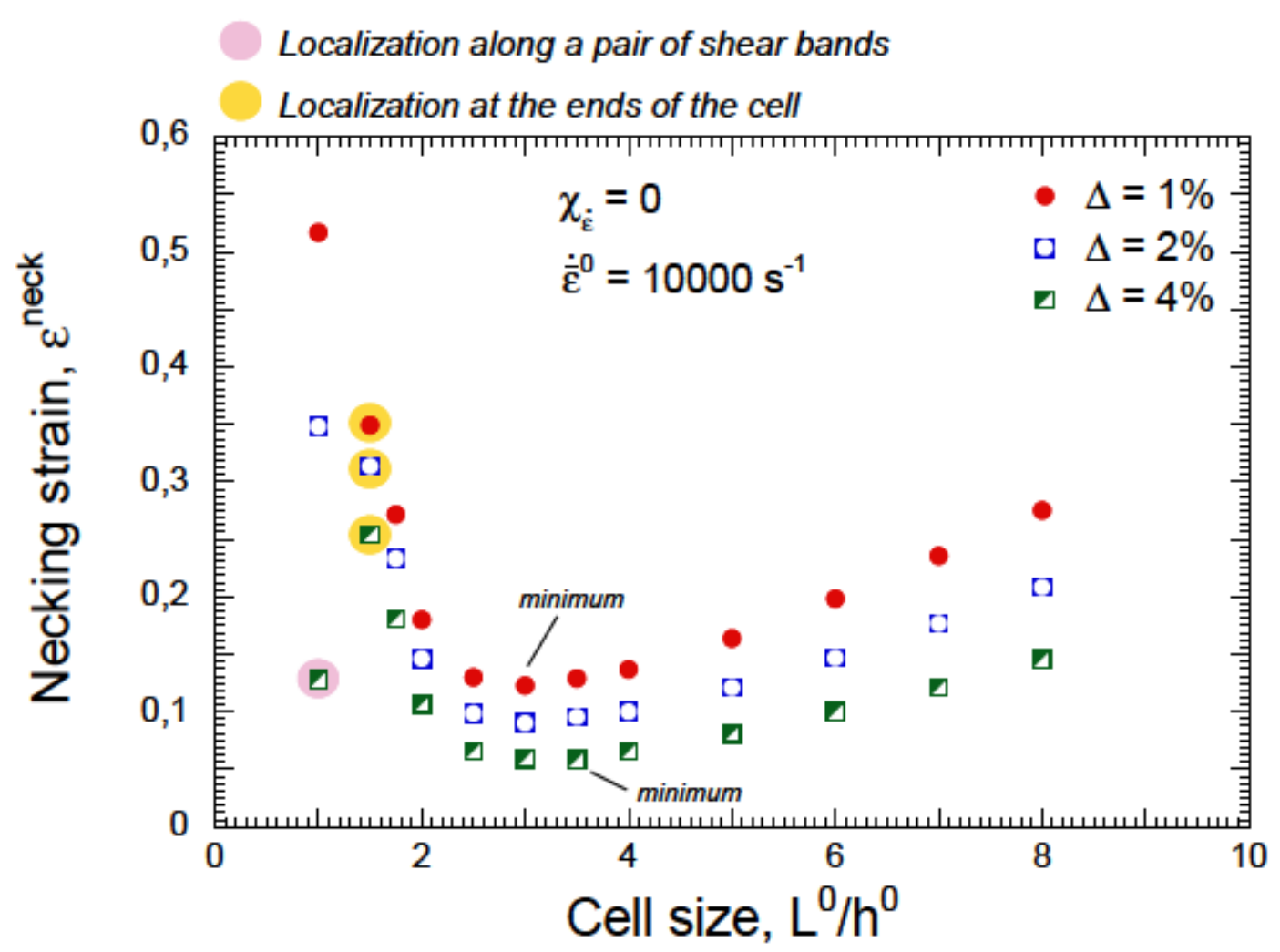

Figure 6: Unitary cell calculations. Necking strain $\varepsilon^{\text {neck }}$ versus initial cell size $L^{0} / h^{0}$ for different values of the imperfection amplitude $\Delta=1 \%, \Delta=2 \%$ (reference) and $\Delta=4 \%$. Reference loading path $\chi_{\dot{\varepsilon}}=0$ and initial loading rate $\dot{\varepsilon}^{0}=10000 \mathrm{~s}^{-1}$.

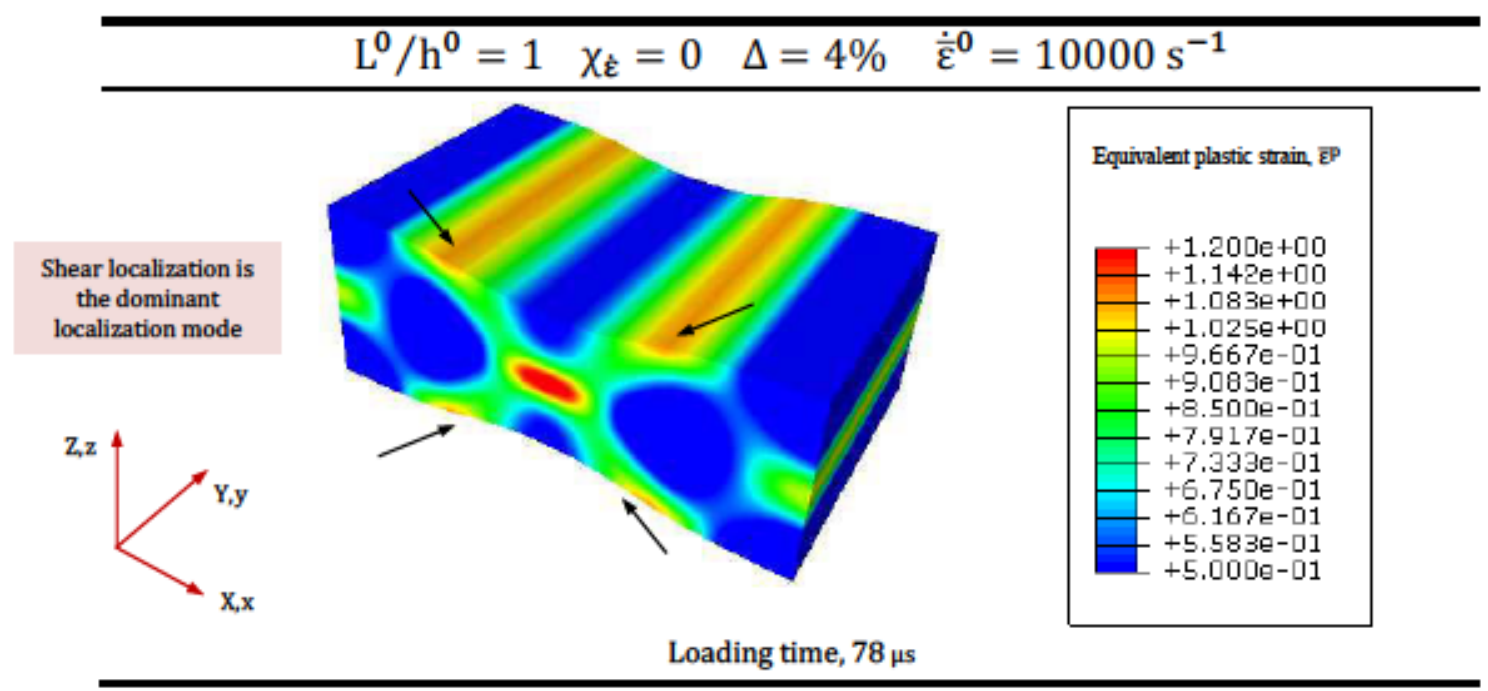

Figure 7: Unitary cell calculations. Contours of equivalent plastic strain $\varepsilon^{p}$ for cell size $L^{0} / h^{0}=1$, loading path $\chi_{\dot{\varepsilon}}=0$, imperfection amplitude $\Delta=4 \%$ and initial loading rate $\dot{\varepsilon}^{0}=10000 \mathrm{~s}^{-1}$. 
size $L^{0} / h^{0}$ for different values of the initial loading rate $\dot{\varepsilon}^{0}=5000 \mathrm{~s}^{-1}, \dot{\varepsilon}^{0}=10000 \mathrm{~s}^{-1}$ (reference), $\dot{\bar{\varepsilon}}^{0}=20000 \mathrm{~s}^{-1}$ and $\dot{\bar{\varepsilon}}^{0}=50000 \mathrm{~s}^{-1}$. The reference combination of loading path and imperfection amplitude is selected.

The necking strain shows a significant increase with the loading rate. This rise is emphasized as the ratio $L^{0} / h^{0}$ increases, since inertia (via strain rate, see Eq. (9)) particularly contributes to the stabilization of long wavelengths (Molinari et al., 2014).

Moreover, the cell length corresponding to the minimum necking strain decreases with loading rate running to $L^{0} / h^{0} \approx 2$ for $\dot{\varepsilon}^{0}=50000 \mathrm{~s}^{-1}$. Further, the minimum of the curve $\varepsilon^{\text {neck }}-L^{0} / h^{0}$ is more pronounced as the loading rate increases. These results suggest that as the role of inertia in the loading process becomes more dominant the necking pattern will be more regular and the neck spacing shorter. The conclusions derived from our unitary cell calculations agree with the experimental and theoretical considerations reported by Mercier et al. (2010) and Mercier and Molinari (2003) who developed specific investigations to demonstrate the damping effect of inertia on ductile materials subjected to plane strain extension. They showed that as the loading rate increases the contribution of inertia takes a dominant role in the localization process leading to the emergence of regularly spaced necks with similar rates of growth. We further elaborate on this specific question in section 9 .

The profound effect that inertia has on the neck spacing is clearly revealed, for instance, in the case of $L^{0} / h^{0}=8$ and $\dot{\varepsilon}^{0}=20000 \mathrm{~s}^{-1}$ for which the localization pattern consists of multiple necks located all along the cell, Fig. 9. This behaviour occurs because the imperfection is largely smoothed during loading (it is not extinguished, but it is largely smoothed) and the cross section of the cell becomes rather constant. Then, with little influence of the geometrical imperfection, the material is prone to develop a neck spacing which requires less investment of energy than the neck spacing corresponding to the cell length. 


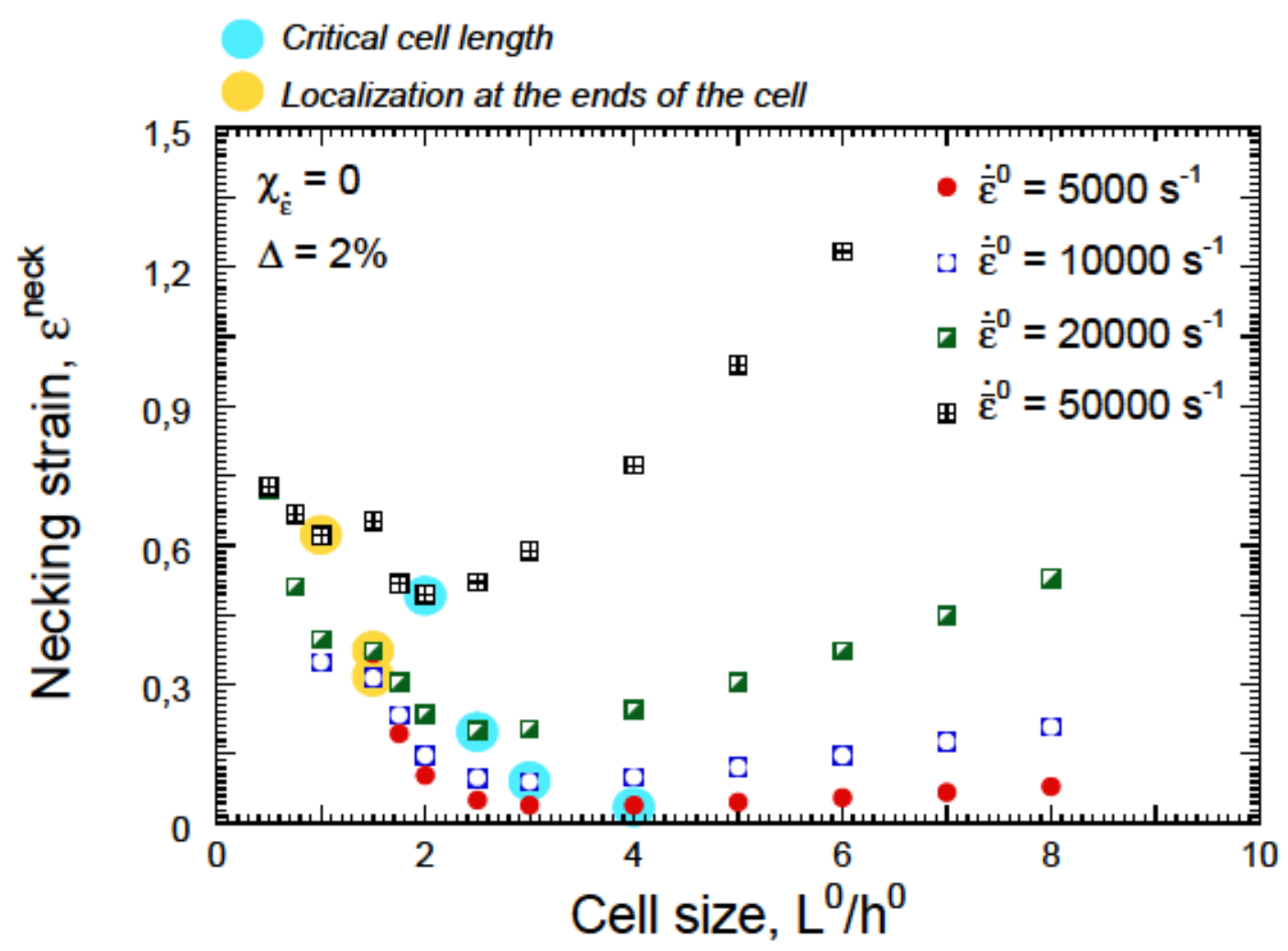

Figure 8: Unitary cell calculations. Necking strain $\varepsilon^{\text {neck }}$ versus initial cell size $L^{0} / h^{0}$ for different values of the initial loading rate $\dot{\varepsilon}^{0}=5000 \mathrm{~s}^{-1}, \dot{\varepsilon}^{0}=10000 \mathrm{~s}^{-1}$ (reference), $\dot{\varepsilon}^{0}=20000 \mathrm{~s}^{-1}$ and $\dot{\varepsilon}^{0}=50000 \mathrm{~s}^{-1}$. Reference loading path $\chi_{\dot{\varepsilon}}=0$ and imperfection amplitude $\Delta=2 \%$.

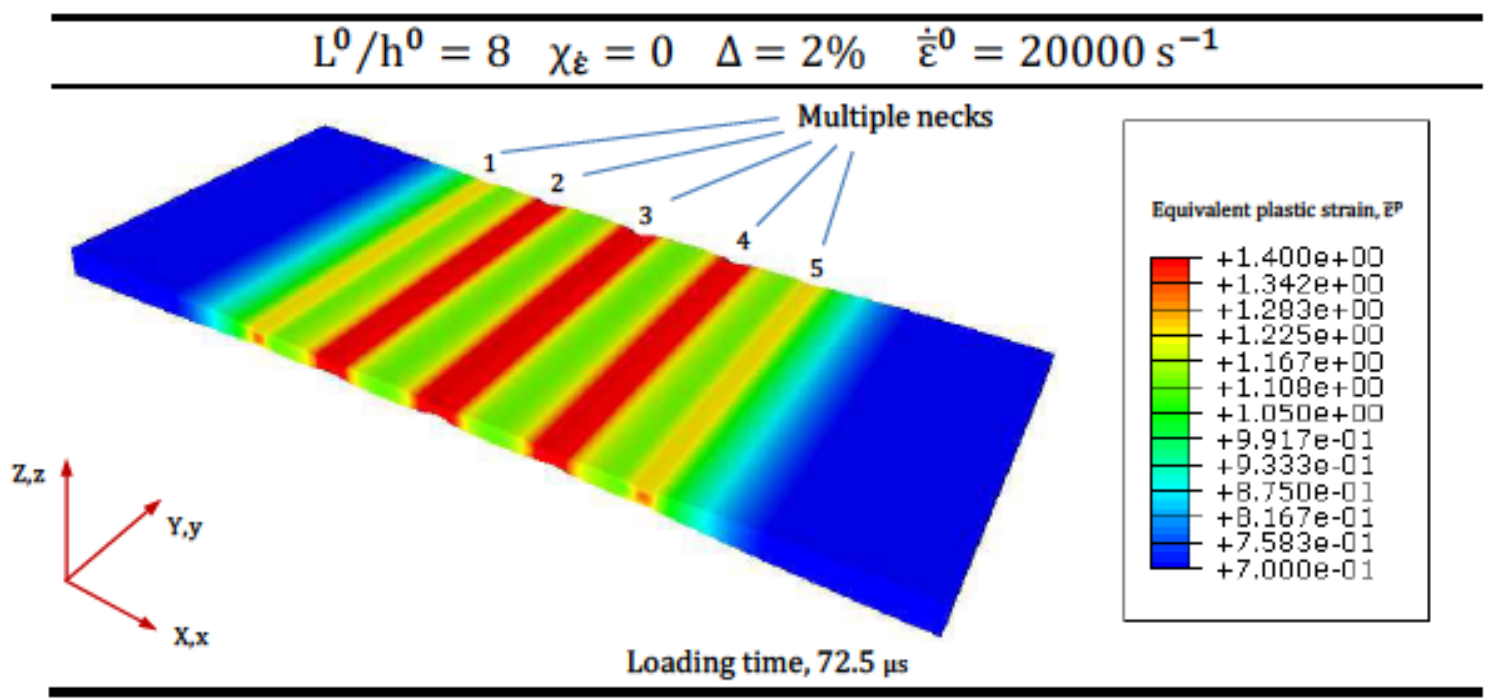

Figure 9: Unitary cell calculations. Contours of equivalent plastic strain $\varepsilon^{p}$ for cell size $L^{0} / h^{0}=8$, loading path $\chi_{\dot{\varepsilon}}=0$, imperfection amplitude $\Delta=2 \%$ (unit cell calculations) and initial loading rate $\dot{\varepsilon}^{0}=20000 s^{-1}$. 


\section{Analysis and results: the interplay between loading path and critical cell size}

In this section, relying on the unitary cell calculations and the linear stability analysis, we uncover the interplay between the loading path and the critical cell size. This is a salient feature of this paper which, from the authors' knowledge, has not been addressed before.

Fig. 10(a) shows the necking strain $\varepsilon^{\text {neck }}$ versus the initial cell size $L^{0} / h^{0}$ for different loading paths $\chi_{\dot{\varepsilon}}=0$ (reference), $\chi_{\dot{\varepsilon}}=0.25, \chi_{\dot{\varepsilon}}=0.5$ and $\chi_{\dot{\varepsilon}}=0.75$. The reference combination of imperfection amplitude and initial loading rate is selected. It is shown that as the parameter $\chi_{\dot{\varepsilon}}$ increases the whole $\varepsilon^{\text {neck }}-L^{0} / h^{0}$ curve is shifted upwards. This means that the material becomes more stable as we move away from plane strain, in agreement with the experimental evidences and the theoretical considerations reported by Verleysen et al. (2011) and Zaera et al. (2015) who analysed the effect of loading path on necking inception at high strain rates. Moreover, the cell size corresponding to the minimum necking strain is shifted to larger values of $L^{0} / h^{0}$ as the loading parameter $\chi_{\dot{\varepsilon}}$ increases. This is because the interplay between inertia and stress multiaxiality leads to greater values of the critical wavelength as the loading path parameter $\chi_{\dot{\varepsilon}}$ increases. This finding agrees with the numerical results obtained by Zaera et al. (2015) who reported a monotonic increase of the neck spacing with the increase of $\chi_{\dot{\varepsilon}}$.

Moreover, Fig. 10(b) shows the normalized necking strain $\bar{\varepsilon}^{\text {neck }}$ versus the normalized initial cell size $\bar{L}^{0} / \bar{h}^{0}$ for $\chi_{\dot{\varepsilon}}=0$ (reference), $\chi_{\dot{\varepsilon}}=0.25, \chi_{\dot{\varepsilon}}=0.5$ and $\chi_{\dot{\varepsilon}}=0.75$. The reference combination of imperfection amplitude and initial loading rate is selected. For each loading path, we have calculated the normalized necking strain as the ratio between $\varepsilon^{\text {neck }}$ and the minimum necking strain. Similarly, the normalized cell size has been calculated as the ratio between $L^{0} / h^{0}$ and the critical cell size. This plot brings to light that the minimum of the $\bar{\varepsilon}^{\text {neck }}-\bar{L}^{0} / \bar{h}^{0}$ curve is weaker as the parameter $\chi_{\dot{\varepsilon}}$ increases. This result suggests that the critical wavelength becomes less prevailing as we move away from plane strain, i.e. the neck spacing shall be less regular as $\chi_{\dot{\varepsilon}}$ increases. This is a key point of our research that is further investigated in section 9 .

Further, in Fig. 11 we compare the critical cell size $\left(L^{0} / h^{0}\right)_{c}$ obtained from our unitary 


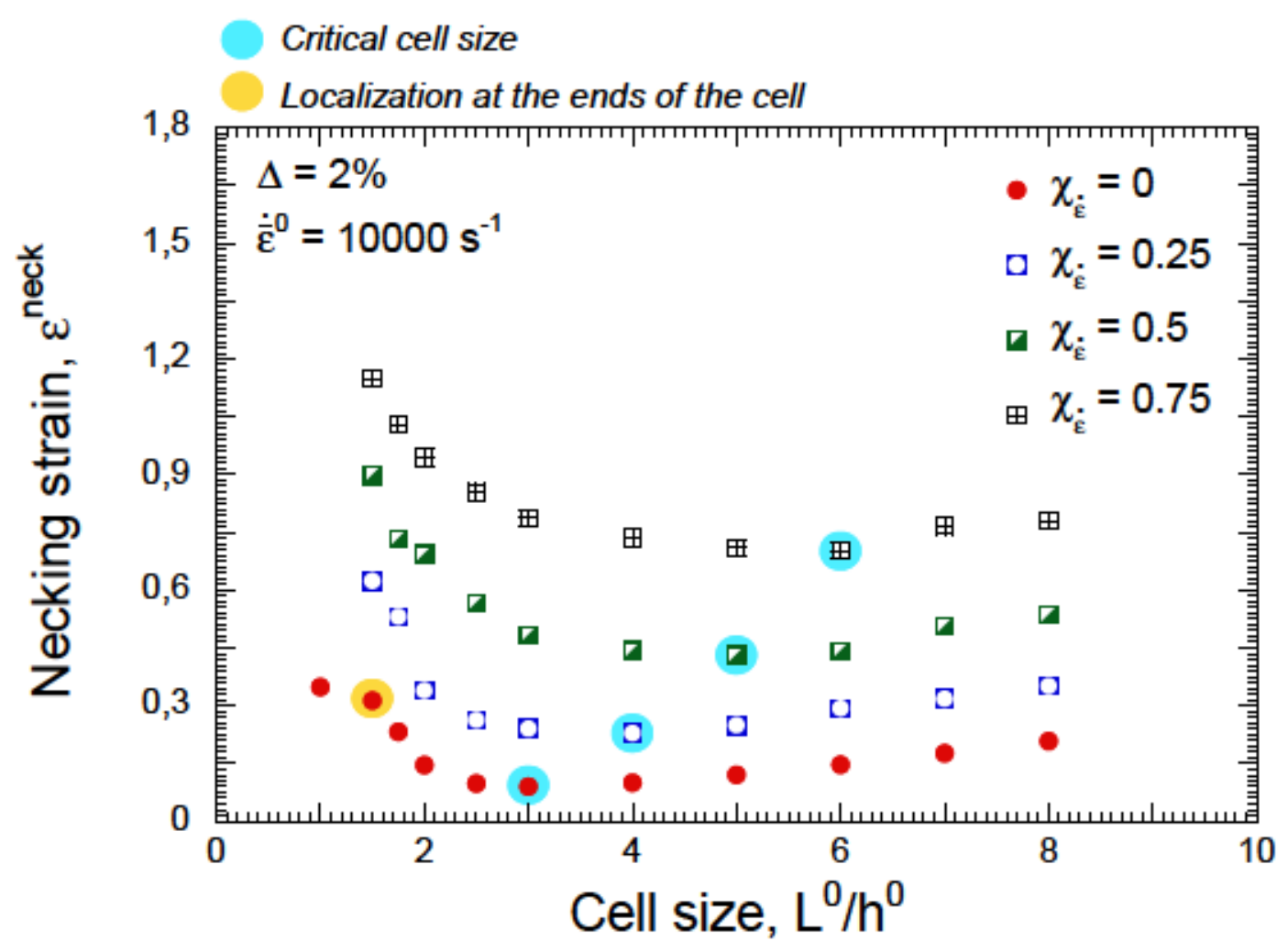

(a)

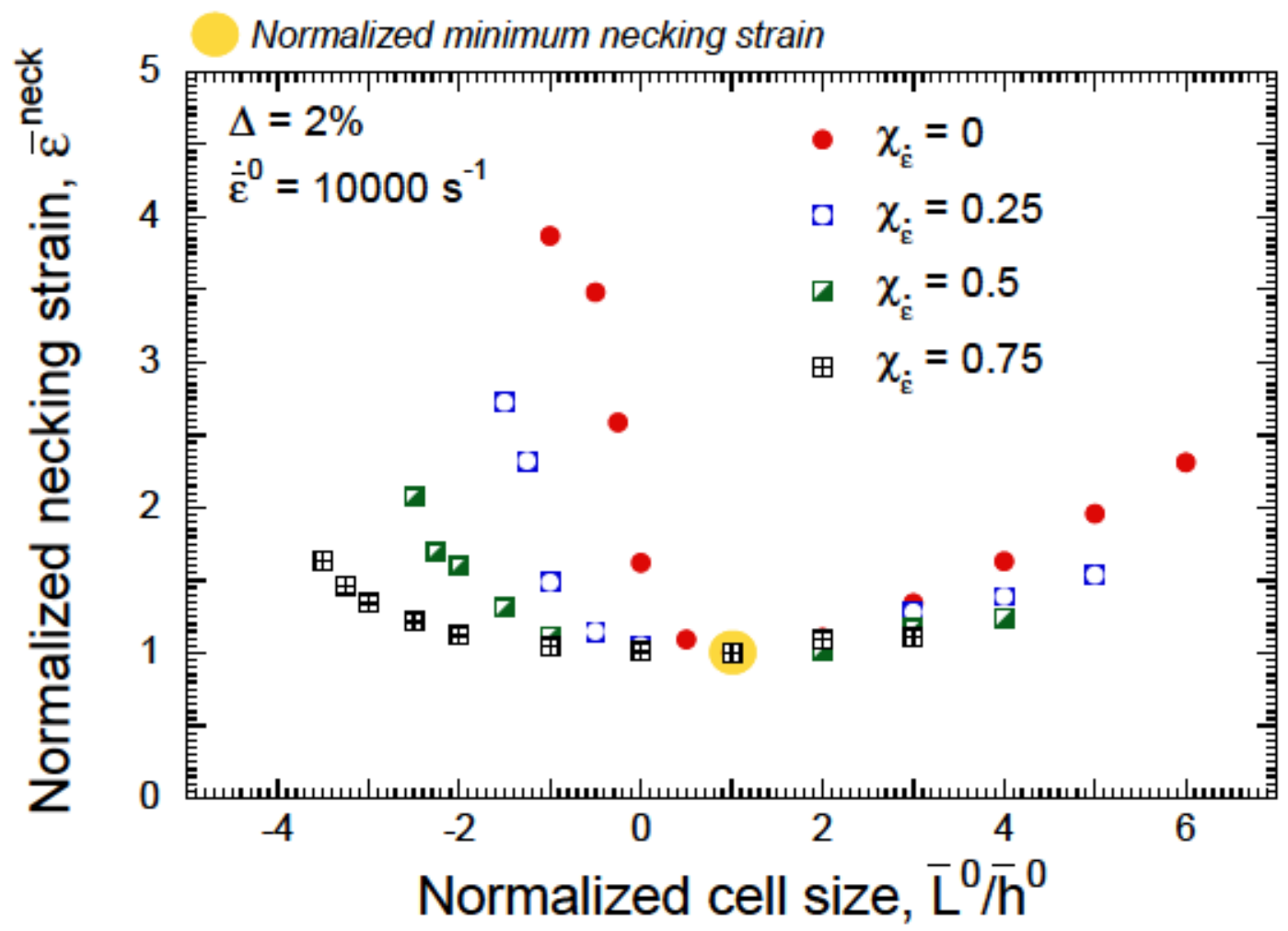

(b)

Figure 10: Unitary cell calculations. (a) Necking strain $\varepsilon^{\text {neck }}$ versus initial cell size $L^{0} / h^{0}$ and (b) normalized necking strain $\varepsilon^{\text {neck }}$ versus normalized cell size $\bar{L}^{0} / \bar{h}^{0}$ for different loading paths $\chi_{\dot{\varepsilon}}=0$ (reference), $\chi_{\dot{\varepsilon}}=0.25, \chi_{\dot{\varepsilon}}=0.5$ and $\chi_{\dot{\varepsilon}}=0.75$. Reference imperfection amplitude $\Delta=2 \%$ and initial loading rate $\dot{\varepsilon}^{0}=10000 s^{-1}$. 
cell calculations (blue shaded points in Fig. 10(a)) with the predictions of the linear stability analysis. In order to obtain the critical cell size from the stability analysis we have considered, relying on the lack of strain hardening of our material (see Eq. (6)), that the instability process (onset of diffuse necking) starts at $t^{1}=0$ (see Eq. (7)). Moreover, as discussed by Rodríguez-Martínez et al. (2013a), the selection of $t^{1}=0$ as the onset of diffuse necking is consistent with the high level of the dimensionless critical perturbation grow $\bar{\eta}_{c}^{+}=\frac{\eta_{c}^{+}}{\dot{\vec{\varepsilon}}}$ (always above 3 ) observed for all the values of $\chi_{\dot{\varepsilon}}$ considered. Then, following the procedure described in section 3 , we calculate the critical wavenumber $\xi_{c}^{\mathscr{L}}$ as a function of $\chi_{\dot{\varepsilon}}$. We associate to this critical wavenumber a prevailing neck spacing:

$L_{c}^{0}=\frac{2 \pi}{\xi_{c}^{\mathscr{Q}}}$. The critical cell size $\left(L^{0} / h^{0}\right)_{c}$ obtained from the linear stability analysis shows very good agreement with the predictions of the unitary cell calculations, see Fig. 11.

On the one hand this comparison shows that there is a critical wavelength which plays a key role in the neck spacing at high strain rates, on the other hand it becomes clear that this critical wavelength increases with the increase of the loading path parameter $\chi_{\dot{\varepsilon}}$. In line with previous works of the authors (e.g. Fressengeas and Molinari $(1985,1994)$; Molinari (1997); Mercier and Molinari (2003, 2004); Rodríguez-Martínez et al. (2013a); Zaera et al. (2014)), these results should be understood as an additional evidence of the ability of the linear stability analysis to predict and rationalize fundamental aspects which control flow localization in ductile materials subjected to dynamic loading.

\section{Analysis and results: the effect of loading path and loading rate on the necking pattern}

In this section, relying on unitary cell and entire plate calculations, we explore the effect of loading path and loading rate on the necking pattern.

Fig. 12 shows results obtained from numerical simulations conducted using the entire plate model for $\chi_{\dot{\varepsilon}}=0$ and $\chi_{\dot{\varepsilon}}=0.25$. The initial loading rate is $\dot{\varepsilon}^{0}=10000 \mathrm{~s}^{-1}$. On the one hand we show contours of equivalent plastic strain $\bar{\varepsilon}^{p}$ which illustrate the emergence of multiple necks aligned with the $Y$ axis. On the other hand we show the equivalent plastic strain $\bar{\varepsilon}^{p}$ versus the normalized plate coordinate $\bar{X}=\frac{X}{L^{0}}$ for different loading times. These 


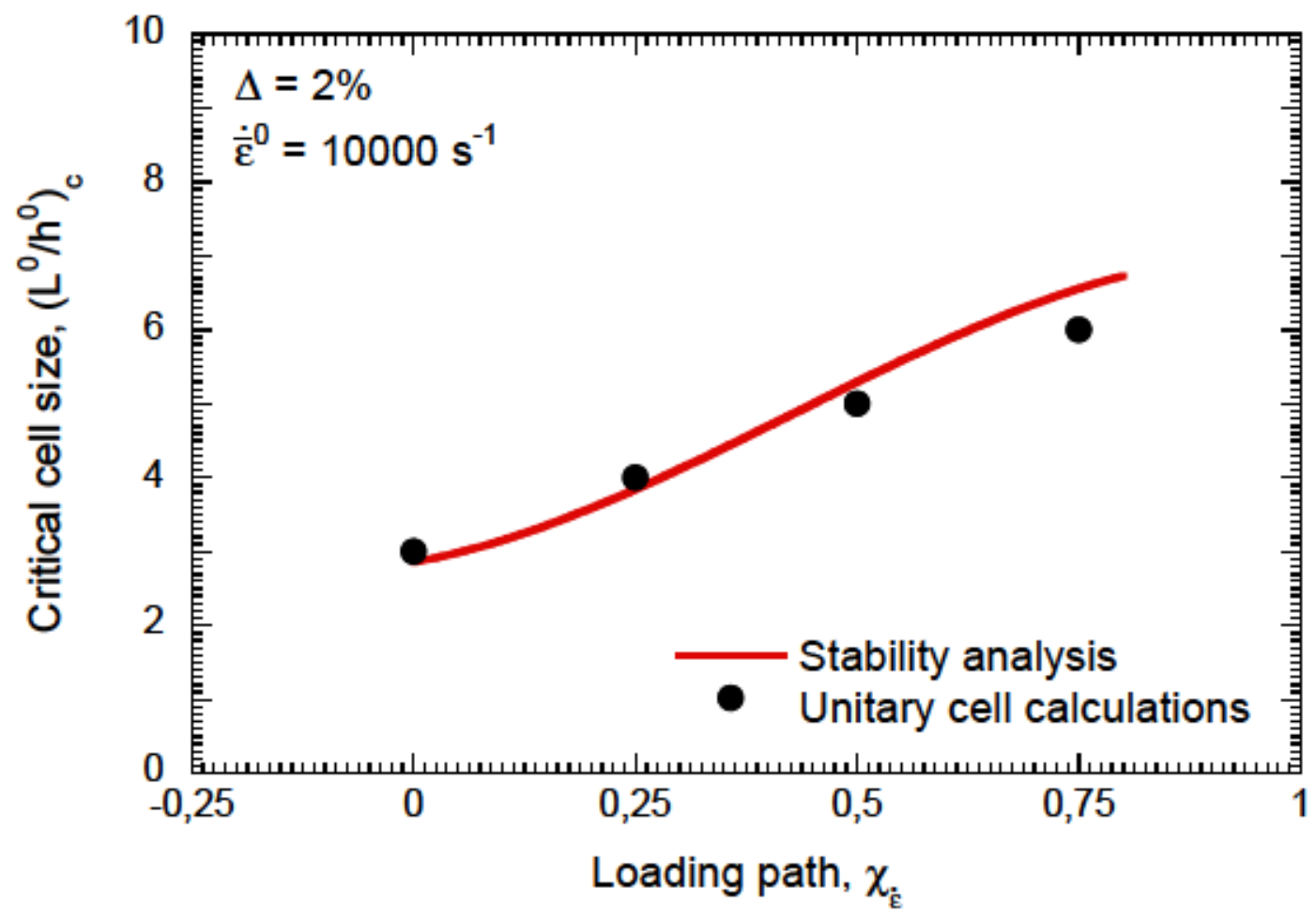

Figure 11: Critical initial cell size $\left(L^{0} / h^{0}\right)_{c}$ versus loading path $\chi_{\dot{\varepsilon}}$. Comparison between linear stability analysis and unitary cell calculations (with the reference imperfection amplitude $\Delta=2 \%$ ). Initial loading rate $\dot{\varepsilon}^{0}=10000 \mathrm{~s}^{-1}$. 
results are enlightening:

1. The number of necks is larger for $\chi_{\dot{\varepsilon}}=0$ than for $\chi_{\dot{\varepsilon}}=0.25$. The average (Lagrangian) normalized neck spacing is $\approx 2.2$ for $\chi_{\dot{\varepsilon}}=0$ and $\approx 2.5$ for $\chi_{\dot{\varepsilon}}=0.25$. The trends find satisfactory agreement with the results reported in Fig. 10(a). It becomes clear that the Lagrangian neck spacing increases with the loading parameter $\chi_{\dot{\varepsilon}}$. The difference with the values found in the unitary cell calculations, $\left(L^{0} / h^{0}\right)_{c}=3$ for $\chi_{\dot{\varepsilon}}=0$ and $\left(L^{0} / h^{0}\right)_{c}=4$ for $\chi_{\dot{\varepsilon}}=0.25$, comes from the fact that the necking strains in the entire plate simulations are larger than those registered in the unitary cell calculations for the same loading conditions (see comments below for Fig. 13). This is because the geometrical perturbations included in the unitary cell calculations affect the necking process to a greater extent than the numerical errors which trigger localization in the entire plate simulations. Decreasing the amplitude of the defect in the unitary cell calculations will provide values of the critical cell size closer to the average neck spacing obtained in the entire plate simulations.

2. The neck spacing is more regular for $\chi_{\dot{\varepsilon}}=0$ than for $\chi_{\dot{\varepsilon}}=0.25$. This result is in agreement with the unitary cell calculations reported in Fig. 10(b). It appeared in Fig. 10(b) that the minimum of the curve $\varepsilon^{\text {neck }}-L^{0} / h^{0}$ is sharper as $\chi_{\dot{\varepsilon}}$ decreases, thus demonstrating that the dispersion observed in neck spacing has to be tighter for small values of $\chi_{\dot{\varepsilon}}$ (i.e. more regular neck spacing). This is a key result of our research that seems to confirm that the critical wavelength which controls the localization process at high strain rates becomes less prevailing as the parameter $\chi_{\dot{\varepsilon}}$ increases. Therefore, the proportion of necks that will develop into fracture sites increases as we approach plane strain. In other words, the proportion of arrested necks increases as we move away from plane strain. Indeed, it is apparent that the occurrence of neck arrest by unloading waves is increased when the neck spacing is less uniform. Theoretically, a perfectly periodic distribution of identical necks would lead to an identical distribution of fracture sites. By contrast, in the case of a non-uniform neck distribution, some necks could be early arrested by unloading waves emanating from dominant localization sites. Then, fracture only occurs at the sites of dominant necks (Mott, 1947; Grady, 1981; Kipp and Grady, 1985; Grady and Olsen, 2003). A 
thorough discussion of the role of defects and unloading waves on the proportion of arrested necks can be found in Molinari et al. (2014).

Previous results show the effect of the loading path on the flow localization pattern. Specifically, we have shown that the necking pattern and the neck spacing become less uniform as $\chi_{\dot{\varepsilon}}$ increases. Next, we explore the role played by the strain rate on the flow localization pattern. 





Fig. 13 shows results obtained from unitary and entire plate calculations. We have explored initial loading rates which range from $5 \cdot 10^{3} \mathrm{~s}^{-1}$ to $5 \cdot 10^{4} \mathrm{~s}^{-1}$. Two different loading paths are investigated: $\chi_{\dot{\varepsilon}}=0$ (reference) and $\chi_{\dot{\varepsilon}}=0.25$. In the unitary cell calculations we have taken the critical cell size and the reference imperfection amplitude $\Delta=2 \%$.

Fig. 13(a) shows the necking strain $\varepsilon^{\text {neck }}$ versus initial strain rate $\dot{\varepsilon}^{0}$. As anticipated in previous paragraphs, the values of $\varepsilon^{\text {neck }}$ are smaller in the case of the unitary cell calculations due to effect of the geometric perturbation included in the model (see Fig. 6). This is a clear illustration of the role of defects' amplitude in the necking strain. It is apparent that the magnitude of the numerical perturbations which trigger localization in the entire plate calculations is smaller than the geometric imperfection included in the unitary cell model. Note that (irrespective of the initial strain rate) the necking strain is larger for $\chi_{\dot{\varepsilon}}=0.25$ than for $\chi_{\dot{\varepsilon}}=0$. Furthermore, the $\varepsilon^{\text {neck }}-\dot{\varepsilon}^{0}$ curves corresponding to $\chi_{\dot{\varepsilon}}=0.25$ run practically parallel to those obtained for $\chi_{\dot{\varepsilon}}=0$.

Moreover, we observe that the necking strain increases with the loading rate which brings to light the neck retardation caused by inertia (Xue et al. (2008)). The relationship between necking strain and initial strain rate for unitary cell and entire plate calculations is very similar. This confirms that the main trends reported in this paper are rather insensitive to the amplitude of the defects which trigger localization, provided that those defects are sufficiently small. The quantification of the neck retardation with increasing strain rate, and therefore of the inertia effects, is addressed using two different measures:

- Absolute measure: we rely on the difference between the necking strains corresponding to the greater and the smaller strain rates investigated, i.e. $\varepsilon_{5 \cdot 10^{4} s^{-1}}^{\text {neck }}-\varepsilon_{5 \cdot 10^{3} s^{-1}}^{\text {neck }}$. Since the $\varepsilon^{\text {neck }}-\dot{\varepsilon}^{0}$ curves for $\chi_{\dot{\varepsilon}}=0$ and $\chi_{\dot{\varepsilon}}=0.25$ are virtually parallel, this measure suggests that the role played by inertia effects on necking localization is largely independent of the loading path.

- Relative measure: we rely on the normalized difference between the necking strains corresponding to the greater and the smaller strain rates investigated $\frac{\varepsilon_{s \cdot 10^{4} s-1}^{\text {neck }}-\varepsilon_{\mathrm{s}-10^{3} s}^{\text {neck }} \mathrm{s}^{-1}}{\varepsilon_{\mathrm{s}-10^{3}}^{\text {nect }} s^{-1}}$. Since the $\varepsilon^{\text {neck }}-\dot{\varepsilon}^{0}$ curves for $\chi_{\dot{\varepsilon}}=0$ and $\chi_{\dot{\varepsilon}}=0.25$ are virtually parallel, the relative 
measure (contrary to the absolute measure) suggests that the role played by inertia effects on necking localization decreases as we move from plane strain to biaxial loading.

Form previous analysis it becomes apparent that to provide a precise idea about the interplay between loading path and inertia effects is a complicated task that still needs further investigation. In this regard we should note that, at least, our analysis rules out that the role played by inertia in flow localization increases with the parameter $\chi_{\dot{\varepsilon}}$.

Fig. 13(b) shows the critical cell size (unitary cell calculations) and the average Lagrangian neck spacing (entire plate calculations) versus the initial strain rate $\frac{\dot{\varepsilon}}{\bar{\varepsilon}}$. As anticipated in previous paragraphs, the values of $\left(L^{0} / h^{0}\right)_{c}$ are greater in the case of the unitary cell calculations due to effect of the geometric perturbation. Moreover note that, irrespective of the initial strain rate, the critical cell size / neck spacing is larger for $\chi_{\dot{\varepsilon}}=0.25$ than

for $\chi_{\dot{\varepsilon}}=0$. Furthermore, the $\left(L^{0} / h^{0}\right)_{c}-\dot{\bar{\varepsilon}}^{0}$ curves obtained for $\chi_{\dot{\varepsilon}}=0$ and $\chi_{\dot{\varepsilon}}=0.25$ run practically parallel. We also observe that the critical cell size and the neck spacing decrease with the strain rate. The latter behaviour is caused by inertia effects, as anticipated in section 7 .

\section{Summary and conclusions}

In this paper we have studied necking localization in ductile plates subjected to dynamic biaxial loading. For that task we have used a 2D linear stability analysis and fully 3D finite element calculations. The linear stability analysis was developed by Zaera et al. (2015) and includes specific features to account for inertia and stress triaxiality effects inside the necking. The finite element computations are conducted using two different models: (1) a unitary cell in which the localization is favoured by a sinusoidal geometrical perturbation and (2) a plate with constant cross section which allows to asses the collective behaviour of multiple necks. A key feature of this investigation is that we have explored a wide spectrum of loading paths ranging from plane strain to (almost) biaxial stretching. Below, we summarize the main outcomes of this work: 


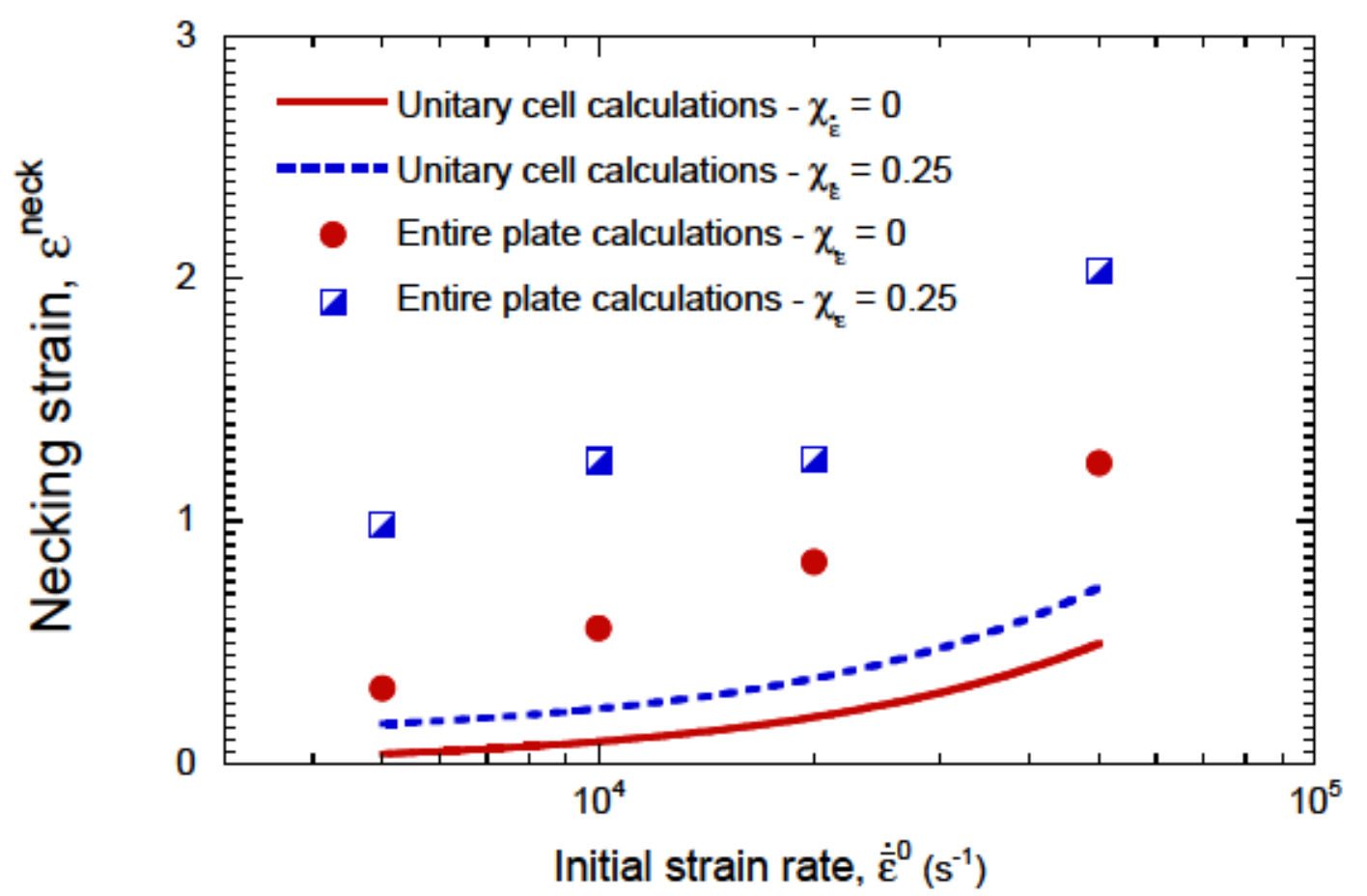

(a)

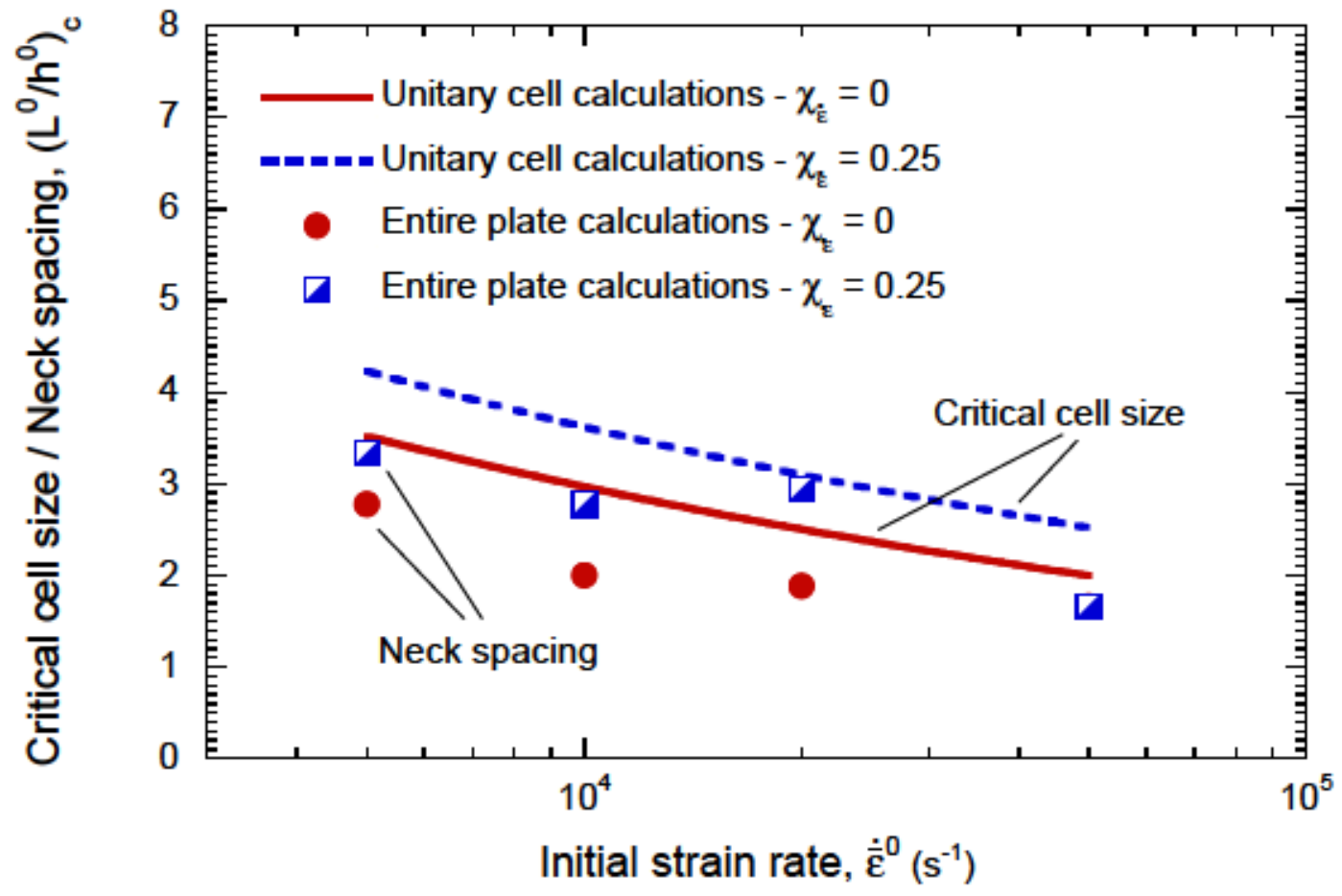

(b)

Figure 13: Unitary cell and entire plate calculations. (a) Necking strain $\varepsilon^{\text {neck }}$ versus initial strain rate $\dot{\varepsilon}^{0}$ and (b) critical cell size/neck spacing $\left(L^{0} / h^{0}\right)_{c}$ versus initial strain rate $\dot{\varepsilon}^{0}$ (lengths are measured in the initial configuration). Two different loading paths are investigated: $\chi_{\dot{\varepsilon}}=0$ (reference) and $\chi_{\dot{\varepsilon}}=0.25$. In the unitary cell calculations we have taken the reference imperfection amplitude $\Delta=2 \%$. 
- We have run unitary cell calculations using various cell sizes in order to show the emergence of a critical wavelength which characterizes the neck spacing at high strain rates. This critical wavelength, which defines the minimum investment of energy required to trigger the neck, is promoted because short wavelengths are stabilized by stress multiaxiality effects and long wavelengths by inertia.

- Unitary cell computations conducted for different loading rates have shown the stabilizing effect of inertia on neck localization. As the role played by inertia on the loading process increases the deterministic character of the necking pattern becomes more significant. It is suggested that at sufficiently high strain rates the geometrical defects may play a secondary role in the necking pattern of viscoplastic shells subjected to impulsive loading.

- Unitary cell and entire plate calculations performed for different loading paths have shown that the neck spacing increases as we move away from plane strain to biaxial stretching. This is because the interplay between inertia and stress multiaxiality leads to greater values of the critical wavelength as the loading path parameter $\chi_{\dot{\varepsilon}}$ increases. This finding is an original outcome of this investigation that, from the authors' knowledge, has not been previously addressed in the literature. It has to be highlighted that the values of the neck spacing obtained from the numerical computations find satisfactory agreement with the predictions of the linear stability analysis. This agreement should be understood as an additional evidence of the ability of the linear stability analysis to capture fundamental aspects which control flow localization in ductile materials subjected to dynamic loading.

- Entire plate calculations performed for different loading paths have revealed that the necking pattern becomes less regular as we move away from plane strain to biaxial stretching. This is because the critical wavelength which controls the localization process at high strain rates becomes less prevailing as the parameter $\chi_{\dot{\varepsilon}}$ increases, as deduced from the unitary cell calculations. This is an original outcome of this investigation which has not been reported before in the literature.

All in all, the combination of analytical and numerical approaches shows that there is 
a critical wavelength which plays a key role in the necking pattern of plates subjected to dynamic biaxial loading when very high strain rates are considered. The salient feature of this paper was to shed light into the close connection between this critical wavelength and the loading path.

\section{Acknowledgements}

JARM, GV, RZ and JFS are indebted to the Ministerio de Economía y Competitividad de España (Projects EUIN2015-62556 and DPI2014-57989-P) for the financial support which permitted to conduct part of this work.

JARM and AM acknowledge the support by the French State through the program Investment in the future operated by the National Research Agency (ANR) and referenced by ANR-11-LABX-0008-01 (LabEx DAMAS).

The research leading to these results has received funding from the European Union's Horizon2020 Programme (Excellent Science, Marie-Sklodowska-Curie Actions) under REA grant agreement 675602 (Project OUTCOME).

\section{References}

Becker, R., 2002. Ring fragmentation predictions using the Gurson model with material stability conditions as failure criterion. International Journal of Solids and Structures $39,3555-3580$.

De Vuyst, T., Vignjevic, R., 2013. Total Lagrangian SPH modelling of necking and fracture in electromagnetically driven rings. International Journal of Fracture 180, 53-70.

Dudzinski, D., Molinari, A., 1988. Instability of visco-plastic deformation in biaxial loading. Comptes Rendues Academie Science Paris 307, 1315-1321.

Dudzinski, D., Molinari, A., 1991. Perturbation analysis of thermoviscoplastic instabilities in biaxial loading. International Journal of Solids and Structures 27, 601-628. 
El Maï, S., Mercier, S., Petit, J., Molinari, A., 2014. An extension of the linear stability analysis for the prediction of multiple necking during dynamic extension of round bar. International Journal of Solids and Structures 51, 3491-3507.

Fressengeas, C., Molinari, A., 1985. Inertia and thermal effects on the localization of plastic flow. Acta Metallurgica 33, 387-396.

Fressengeas, C., Molinari, A., 1994. Fragmentation of rapidly stretching sheets. European Journal of Mechanics A/Solids 13, 251-268.

Goto, D., Becker, R., Orzechowski, T., Springer, H., Sunwoo, A., Syn, C., 2008. Investigation of the fracture and fragmentation of explosively driven rings and cylinders. International Journal of Impact Engineering 35, 1547-1556.

Grady, D., 1981. Fragmentation of solids under impulsive stress loading. Journal of Geophysical Research 86, 1047-1054.

Grady, D., 2002. Fragmentation of expanding cylinders and the statistical theory of N. F. Mott. In: Furnish, M. D., Thadhani, N. N., Horie, Y. (Eds.), Shock Compression of Condensed Matter - 2001). . American Institute of Physics, pp. 799-802.

Grady, D. E., Olsen, M. L., 2003. A statistics and energy based theory of dynamic fragmentation. International Journal of Impact Engineering 29, 293-306.

Guduru, P. R., Freund, L. B., 2002. The dynamics of multiple neck formation and fragmentation in high rate extension of ductile materials. International Journal of Solids and Structures 39, 5615-5632.

Hiroe, T., Fujiwara, K., Hata, H., Takahashi, H., 2008. Deformation and fragmentation behaviour of exploded metal cylinders and the effects of wall materials, configuration,explosive energy and initiated locations. International Journal of Impact Engineering $35,1578-1586$.

Kipp, M. E., Grady, D. E., 1985. Dynamic fracture growth and interaction in one dimension. Journal of the Mechanics and Physics of Solids 33, 399-415. 
Knoche, P., Needleman, A., 1993. The effect of size on the ductility of dynamically loaded tensile bars. European Journal of Mechanics A/Solids 12, 585-601.

Mercier, S., Granier, N., Molinari, A., Llorca, F., Buy, F., 2010. Multiple necking during the dynamic expansion of hemispherical metallic shells, from experiments to modelling. Journal of the Mechanics and Physics of Solids 58, 955-982.

Mercier, S., Molinari, A., 2003. Predictions of bifurcations and instabilities during dynamic extensions. International Journal of Solids and Structures 40, 1995-2016.

Mercier, S., Molinari, A., 2004. Analysis of multiple necking in rings under rapid radial expansion. International Journal of Impact Engineering 30, 403-419.

Molinari, A., 1997. Collective behaviour and spacing of adiabatic shear bands. Journal of the Mechanics and Physics of Solids 45, 1551-1575.

Molinari, A., Mercier, S., Jacques, N., 2014. Dynamic failure of ductile materials. Procedia IUTAM 10, 201-220.

Molinari, A., Ravichandran, G., 2005. Constitutive modeling of high-strain-rate deformation in metals based on the evolution of an effective microstructural length. Mechanics of Materials 37, 737-7528.

Mott, N. F., 1947. Fragmentation of shell cases. In: . Series A. Proceedings of the Royal Society, London, pp. 300-308.

Needleman, A., 1988. Material rate dependence and mesh sensitivity in localization problems. Computer Methods in Applied Mechanics and Engineering 67, 69-85.

Nemat-Nasser, S., Guo, W., 2000. High strain-rate response of commercially pure vanadium. Mechanics of Materials 32, 243-260.

Ravi-Chandar, K., Triantafyllidis, N., 2015. Dynamic stability of a bar under high loading rate: Response to local perturbations. International Journal of Solids and Structures 58, $301-308$. 
Rodríguez-Martínez, J. A., Vadillo, G., Fernández-Sáez, J., Molinari, A., 2013a. Identification of the critical wavelength responsible for the fragmentation of ductile rings expanding at very high strain rates. Journal of the Mechanics and Physics of Solids 61, $1357-1376$.

Rodríguez-Martínez, J. A., Vadillo, G., Zaera, R., Fernández-Sáez, J., 2013b. On the complete extinction of selected imperfection wavelengths in dynamically expanded ductile rings. Mechanics of Materials 60, 107-120.

Rodríguez-Martínez, J. A., Vadillo, G., Zaera, R., Fernández-Sáez, J., Rittel, D., 2015. An analysis of microstructural and thermal softening effects in dynamic necking. Mechanics of Materials 80B, 298-310.

Rusinek, A., Klepaczko, J. R., 2001. Shear testing of a sheet steel at wide range of strain rates and a constitutive relation with strain-rate and temperature dependence of the flow stress. International Journal of Plasticity 17, 87-115.

Rusinek, A., Zaera, R., 2007. Finite element simulation of steel ring fragmentation under radial expansion. International Journal of Impact Engineering 34, 799-822.

Simulia, 2013. ABAQUS/Explicit User's Manual, version 6.13 Edition. Dassault Systèmes, Providence, USA.

Sørensen, N. J., Freund, L. B., 2000. Unstable neck formation in a ductile ring subjected to impulsive radial loading. International Journal of Solids and Structures 37, 2265-2283.

Triantafyllidis, N., Waldenmyer, J. R., 2004. Onset of necking in electro-magnetically formed rings. J Mech Phys Solids 52, 2127-2148.

Vadillo, G., Rodríguez-Martínez, J. A., Fernández-Sáez, J., 2012. On the interplay between strain rate and strain rate sensitivity on flow localization in the dynamic expansion of ductile rings. International Journal of Solids and Structures 49, 481-491.

Verleysen, P., Peirs, J., Van Slycken, J., Faes, K., Duchene, L., 2011. Effect of strain rate on the forming behaviour of sheet metals. Journal of Materials Processing Technology 211, 1457-1464. 
Voyiadjis, G. Z., Abed, F. H., 2006. A coupled temperature and strain rate dependent yield function for dynamic deformations of bcc metals. International Journal of Plasticity 22, $1398-1431$.

Xue, Z., Vaziri, A., Hutchinson, J., 2008. Material aspects of dynamic neck retardation. Journal of the Mechanics and Physics of Solids 56, 93-113.

Zaera, R., Fernández-Sáez, J., 2006. An implicit consistent algorithm for the integration of thermoviscoplastic constitutive equations in adiabatic conditions and finite deformations. International Journal of Solids and Structures 43, 1594-1612.

Zaera, R., Rodríguez-Martínez, J. A., Vadillo, G., Fernández-Sáez, J., 2014. Dynamic necking in materials with strain induced martensitic transformation. Journal of the Mechanics and Physics of Solids 64, 316-337.

Zaera, R., Rodríguez-Martínez, J. A., Vadillo, G., Fernández-Sáez, J., Molinari, A., 2015. Collective behaviour and spacing of necks in ductile plates subjected to dynamic biaxial loading. Journal of the Mechanics and Physics of Solids. 85, 245-269.

Zhang, H., Ravi-Chandar, K., 2010. On the dynamics of localization and fragmentation-IV. Expansion of Al 6061-O tubes. International Journal of Fracture 163, 41-65.

Zhou, F., Molinari, J. F., Ramesh, K. T., 2006. An elasto-visco-plastic analysis of ductile expanding ring. International Journal of Impact Engineering 33, 880-891. 